\title{
Social identity and risky leisure activities: implications for welfare and policy
}

\section{Andrea Mannberg ${ }^{1}\left[\right.$ Tomas Sjögren $^{2}$}

Received: 2 September 2020 / Accepted: 31 January 2022 / Published online: 22 February 2022

(c) The Author(s) 2022

\begin{abstract}
In this paper, we build on theories in psychology and economics and link positional preferences to private agents' identification with a social group, and the social norms present in that group. The purpose of our paper is to analyze behavioral, welfare, and policy implications of a link between private agents's social identity and a risky leisure activity. Our results suggest that, when the outcome of the positional activity is uncertain, the over-consumption result that is associated with positional preferences in a deterministic framework need not apply to all agents in a social equilibrium. The reason is that agents have incentives to act with caution in order to avoid failure when the outcome of the socially valued activity is uncertain. We also show how policy can be used to improve the welfare within a social group where the risky leisure activity is positional.
\end{abstract}

\section{Introduction}

Social media platforms are filled with posts that display people who voluntarily engage in activities that could have dire consequences. Why do people voluntarily engage in activities that expose them to potentially fatal risk? One possible answer is that accomplishing risky tasks provide agents with social status, i.e., risky activities seem to be positional within some social groups.

The purpose of this paper is to analyze the implications of positional preferences associated with risky leisure activities for individual behavior, group behavior, welfare and policy. We focus on risky leisure activities for four reasons: (1) Risky

Andrea Mannberg

andrea.mannberg@uit.no

Tomas Sjögren

tomas.sjogren@umu.se

1 School of Business and Economics, UiT The Arctic University of Norway, Breivangvegen 23, 9010, Tromsø, Norway

2 Department of Economics, Umeå School of Business, Economics and Statistics, Umeå University, Biblioteksgränd 6, 90187 Umeå, Sweden 
leisure activities are voluntary and not associated with direct monetary benefits. In other words, the agent is not compensated for unwanted risk exposure by a higher salary. (2) Risky leisure activities are associated with potentially large external costs (e.g., via health care, costs for safety arrangements, and rescue actions), (3) The popularity of 'extreme' sports and adventure tourism has increased dramatically during the past few decades. Indeed, the adventure tourism sector had a compound annual growth rate of 21 percent between 2012 and 2018 (Adventure Travel Trade Association, 2018). (4) The rapid development of social media technologies means that leisure activities have become substantially more visible.

Already Veblen (1899) and Duesenberry (1949) argued that agents try to signal status via their behavior. Today there is substantial empirical evidence in support of both this hypothesis and for the related idea that agents experience happiness if they do well in comparison with some reference group(s) (happiness research: e.g. Easterlin 1995; Blanchflower and Oswald 2004; Ferrer-i-Carbonell 2005; Luttmer 2005, questionnaire-based experiments: e.g. Solnick and Hemenway 1998, 2005; Johansson-Stenman et al 2006; Carlsson et al. 2007, psychology; e.g., Festinger 1957; Rivis and Sheeran 2003; Kirkpatrick and Ellis 2007; White et al. 2009, and brain science: Fleissbach et al 2007).

Positional preferences are of interest to economists because they give rise to (potentially large) negative external effects and wasteful use of resources: Alpizar et al. (2005) estimate that 50 per cent of the total utility gain from increased consumption of status goods stem from the increase in relative consumption, and Mujcic and Frijters (2013) find that individuals in their sample of Australian university students demanded a 200 percent compensation in income to accept going from the top to the bottom of the income distribution.

A typical result found in the empirical literature is that visible goods are more positional than less visible goods, and that the consumption of physical goods are more positional than the consumption of leisure (Alpizar et al. 2005; Solnick and Hemenway, 1998; 2005; Carlsson et al. 2007). However, there are several reasons for why it may be erroneous to conclude that people are not positional w.r.t leisure activities. One is that time is a fairly non-visual good, whereas leisure activities are potentially much more visual, especially after the introduction of social media. Another is that, in line with the "strategic handicapping principle" ${ }^{1}$ (Zahavi 1975; Grafen 1990a, b), conspicuous consumption may be a signal about an underlying quality (Boone 1998), and therefore that it is the accomplishment of a difficult task rather than the consumption of time that provides the agent with social status. Third, the type of accomplishments that matter for an individual are likely to depend on the norms present in the social groups to which the individual ascribes membership.

\footnotetext{
${ }^{1}$ This principle states that characteristics and behaviors, associated with high energy costs and/or an increased risk of predation (e.g., the ornate plumage of some birds), have evolved because they signal underlying qualities that increase the survival chances of a species. Individuals who enage in risky displays or behavior are therefore awarded a high social rank, which in turn provides them with both productive and reproductive resources (Boone, 1997).
} 
The last argument suggests that what individuals are positional about depends on their social identities. This is in line with the pioneering work by Akerlof and Kranton (2000), who showed that the desire to become an accepted member in a valued social group may drive people to overinvest (with respect to their ability) in status giving activities. It is also in line with theories in psychology, such as identity theory (Stryker 1968; Stryker and Serpe 1982; Burke and Reitzes 1981; Burke 1991; Stets and Burke 2000) and social identity theory (Tajfel et al. 1971; Tajfel 1982; Turner and Oaks 1986). These theories argue that an individual's sense of personal identity is a unique combination of different sub-identities linked to the macro, meso and micro social groups to which the individual belongs. Identity theories suggest that (i) individuals self-categorize into social groups, (ii) most individuals belong to a plethora of different social groups, and (iii) the types of behaviour and characteristics associated with social status will differ between groups due to the social norms prevalent in each group. Individuals gain self-esteem from their performance relative to other groups and relative to other group members for activities and behaviours that are valued within the social group (Festinger 1957; Rivis and Sheeran 2003; White et al. 2009). The extent to which an individual cares about her relative standing in the social hierarchy depends on her dependency on social affirmation and on how important membership to that specific group is (Hogg 2016). Several studies suggest that there is a link between social status, social comparisons and risk-taking behaviour, e.g., for drug use, and sun tanning (Leary et al. 1994; Aloise-Young et al. 1996; Miller-Johnson et al. 2003; Fischer et al. 2011). Jellison and Riskin (1970) find empirical support for that risk-taking is a signal of ability, and that most individuals want to be higher in abilities in comparison to others (i.e., a positional preference for ability).

The arguments presented above imply that agents can be positional in several dimensions; not only in the consumption/income dimension but also w.r.t. particular leisure activities. The latter observation constitutes the point of departure for this paper. We analyze how positional preferences w.r.t. a risky leisure activity affects the behavior and welfare in a social group where this activity is a central feature of the agents' identity. The below analysis focuses on a leisure activity that is associated with risk of physical injury. Examples include, but are not are not limited to, activities such as mountaineering, backcountry skiing, downhill mountainbiking, scuba diving, parachuting, and long-distance running. In this context, we also analyze how policy can be used to improve the welfare within the social group. To address these issues, we set up a model where the agents have preferences over consumption, pure leisure and of their accomplishments in the leisure activity. Successfully performing a more difficult task provides the agent with a higher utility but there is a risk of failure, which increases with the difficulty level. Each agent's identity is related to the leisure activity and depends on (i) how strongly the agent identifies herself as a, e.g., mountaineer or mountainbiker, and on (ii) how successful the agent perceives herself to be in comparison with other people in the social group.

In the below analysis, we focus on a situation where the social group consists of agents with high respectively low skill in the activity. In our base model, we assume that all agents compare their performance with the observed difficulty level performed by both high and low skilled agents. We thereafter evaluate the special case 
where all agents only compare themselves with the high-skilled group. This special case is consistent with arguments put forward by e.g. Eckerstorfer and Wendner (2013) who (in the context of consumption externalities) argue that it is more realistic to assume that some agents contribute more to the reference level than others. It is also supported by empirical research on positional preferences (Clark and Senik 2010), which find that most people engage in upward comparisons concerning income. Finally, Cowan et al (2004) argue that people, who do not belong to some elite group, aspire to mimic the consumption of the elite (aspiration effect) while people who belong to the elite can use consumption to outshine their peers (distinction effect).

Within this framework, we address the following topics: (i) Is there a qualitative difference in the behavioral response to an increase in the reference level associated with the positional activity when the outcome of the activity is certain compared with when the outcome is uncertain? (ii) Is there a qualitative difference in the behavioral response to an increase in the strength of the agent's positional concern when the outcome of the positional activity is certain compared with when the outcome is uncertain? (iii) Under certainty, the keeping up with the Joneses effect implies that all agents over-consume the positional good in an unregulated equilibrium. Does the over-consumption result also apply when the positional activity is uncertain, or is it possible that some sub-group(s) may under-consume the positional good under uncertainty? (iv) What policy can be used to improve the welfare within a social group where the identity is linked to a risky leisure activity? To address the last question, we proceed along two routes. First, we pose the question of what policy instrument that would be needed to implement the first-best outcome and we show that an optimal design of fees to perform the leisure activity at different difficulty levels will be sufficient. However, a system of difficulty-adjusted fees is likely to be difficult to implement in practice because of the associated monitoring cost. Therefore, we also consider an alternative approach, namely that the risk of physical injuries means that the agent may require assistance (e.g., via rescue operations, or medical care). We show that if assistance is required, then the agent should pay an accompanying fee associated with this assistance. In the final part of the paper, we characterize the optimal design of such an assistance cost function.

This paper makes two distinct contributions to the literature. The first is to relate positional preferences to a risky leisure activity with an uncertain outcome. We show that the standard keeping up with the Joneses effect (i.e. that an increase in the benchmark consumption associated with the comparison group has a positive effect on the individual agent's consumption) may be reversed when the positionality activity is associated with an uncertain outcome. As a result, some people may under-engage in the leisure activity in the resulting equilibrium. This result can be related to the analysis conducted by Bakshi and Chen (1996) who considered the hypothesis that investors accumulate wealth to obtain wealth-induced social status. They found that when investors care about status and about "keeping up with the Joneses," the investors are less prone to take on risk. The second contribution is to show how a policy-maker can use participation fees or assistance fees to improve the welfare in a social group, which engages in a risky leisure activity. We show that a system of non-linear participation fees will be sufficient to implement the first-best 
outcome whereas an optimal design of assistance fees can improve the welfare, albeit not all the way to the first-best level.

The outline of the paper is as follows. In Sect. 2, we characterize the individual agents' decision problem and in Sect. 3 we derive some comparative static results. In Sect. 4, we characterize the equilibrium within the social group and compare this outcome with the first-best outcome. Policy implications are addressed in Sect. 5 and the paper is concluded in Sect. 6.

\section{The agent's decision problem}

Chang (2013) has pointed out that the concept of identity/self-esteem developed by Akerlof and Kranton (2000) can be used to motivate why relative income matters for subjective wellbeing (utility). Therefore, consider an agent who has preferences for consumption, $c$, pure leisure, $z$, and a risky activity. We define pure leisure as the time spent doing no activity at all. The preferences for consumption and pure leisure are captured by the sub-utility functions $u(c)$ and $v(z)$, where $u_{c}, v_{z}>0$ and $u_{c c}, v_{z z} \leq 0$. To keep the model as simple as possible, we assume that the agent is endowed with a fixed income $w$ which means that consumption is given by $c=w$. To model the preference for the risky activity, we assume that the agent faces a continuum of activity related tasks which she can attempt to perform. Each activity/task is associated with a unique difficulty level which is captured by an index $y$ which is monotonously increasing in the difficulty level and distributed over the interval $y \in\left(0, y^{\max }\right)$. Activity $y=0$ has zero probability of failure and is therefore risk-free. An activity with $y>0$ will be labelled as risky because the probability of failure is positive. Activity $y^{\max }$ is the most difficult task and therefore has the lowest probability of success. The subjective wellbeing/pride of successfully performing activity $y$ is captured by the sub-utility function $g(y)$. We assume that this pride function has the following properties:

$$
g(0)=0, g_{y}(y)>0, g_{y y}(y)<0, \lim _{y \rightarrow 0} g_{y}(y) \rightarrow \infty .
$$

If the agent fails in her attempt to perform a risky activity, the pride function takes the value zero.

The agent is part of a social group where all members have preferences for the risky activity. The social group consists of two ability types, 1 and 2, where ability type 2 is more skilled than ability type 1. An agent's skill level affects the probability of successfully accomplishing a given activity $y$. Let $p^{i}(y)$ denote the probability that ability type $i=1,2$ successfully accomplishes activity $y$. We assume that $p^{i}(y)$ satisfies the following properties:

$$
p^{i}(0)=1, p_{y}^{1}(y)<p_{y}^{2}(y)<0, p_{y y}^{i}(y) \leq 0 \text { for } y>0 .
$$

The first assumption implies that there is no risk of failing the risk-free activity while the second assumption implies that the probability of success decreases with the difficulty level, but at a slower rate for the high-skilled type. The latter feature, in turn, ensures that the high-skilled type has a higher probability of successfully 
accomplishing a given risky activity than the low-skilled type, i.e. $p^{2}(y)>p^{1}(y)$ holds for all $y>0$. Finally, by assuming that $p^{i}(y)$ is weakly concave, we incorporate the feature that the probability of success does not decrease at a slower rate as $y$ becomes larger.

An agent of type $i$ derives utility, henceforth referred to as identity, from being a valued member of the social group. A person's identity depends on how successful she is in performing the risky activity in comparison with the other members in the social group. When evaluating her performance, an individual of type $i$ compares the difficulty level of the activity she attempts to perform with a reference level $\bar{y}^{i}$. This reference level is a weighted average of the observed successful accomplishments made by the two ability types in the social group. If we let $y^{i}$ denote the activity that is accomplished by agent type $i$, then agent type $i$ 's reference level is determined by $\bar{y}^{i}=x^{i} y^{1}+\left(1-x^{i}\right) y^{2}$. Here $x^{i} \in[0,1]$ is the weight that an agent of type $i$ attaches to type 1 in the weighted average calculation of $\bar{y}^{i}$. The individual agent is myopic and treats the levels of $y^{1}$ and $y^{2}$ observed within the social group as exogenous in the weighted average calculation of $\bar{y}^{i}$. Agent type $i$ 's relative performance is captured by ${ }^{2} \Delta^{i}=y^{i}-\bar{y}^{i}$ and the agent's identity, $I^{i}$, is a function of this performance measure; $I^{i}=I\left(\Delta^{i}\right)$. This identity function is increasing and concave in $\Delta^{i}$, and we use the normalization $I(0)=0$. For the analysis below, we note that $I_{\bar{y}}^{i}=-I_{y}^{i}<0, I_{\overline{y y}}^{i}=I_{y y}^{i}<0$ and $I_{y \bar{y}}^{i}=-I_{y y}^{i}>0$.

There is a time cost, $h\left(y^{i}\right)$, associated with performing activity $y^{i}$. We assume that this cost function satisfies $h_{y}>0$ and $h_{y y} \geq 0$. The weak convexity reflects that it usually takes more time to prepare for, and to perform, a more difficult activity. Normalizing the time endowment to one, the time constraint can be written as $z^{i}=1-h\left(y^{i}\right)$. The overall utility associated with successfully performing activity $y^{i}$ is specified as follows ${ }^{3}$ :

$$
U^{i}=u(w)+v\left(1-h\left(y^{i}\right)\right)+\beta^{i} g\left(y^{i}\right)+\zeta^{i} I\left(y^{i}-\bar{y}^{i}\right),
$$

where the parameters $\beta^{i}$ and $\zeta^{i}$ reflect how important pride and identity are for the agent's overall utility. Note that $U_{y \bar{y}}^{i}=\zeta^{i} I_{y \bar{y}}^{i}>0$, i.e. the cross-derivative of the utility function w.r.t. the positional activity $(y)$ and the reference level $(\bar{y})$ is positive. By using an analogous definition as Dupor and Liu (2003), ${ }^{4}$ we define $U_{y \bar{y}}^{i}>0$ to imply that the agent has "keeping up with the Joneses" preferences. For the analysis

\footnotetext{
2 This measure is analogous to that used in much of the literature on positional consumption preferences. See e.g. Corneo and Jeanne (1997); Ljungqvist and Uhlig (2000); Aronsson and Johansson-Stenman (2008; 2010).

3 Without loss of generality, we assume that $w$ is the same for both agent types.

${ }^{4}$ In a framework where agents have preferences over private consumption (the positional good), percapita consumption (the reference level) and leisure, Dupor and Liu (2003) define preferences to exhibit "keeping up with the Joneses" if the marginal rate of substitution between leisure and consumption increases with per-capita consumption. If the preferences are additively separable between, on one hand private consumption and per-capita consumption, and on the other hand leisure, Dupor and Liu point out that the definition above is equivalent with assuming that the cross-derivative of the utility function w.r.t. private consumption (the positional good) and per-capita consumption (the reference level) is positive.
} 
below, let us define an agent's degree of positionality w.r.t. successfully performing the risky activity as follows ${ }^{5}$ :

$$
\alpha^{i}=\frac{U_{\Delta}^{i}}{U_{y}^{i}+U_{\Delta}^{i}}=\frac{\zeta^{i} I_{y}^{i}}{\beta^{i} g_{y}^{i}+\zeta I_{y}^{i}} \in[0,1]
$$

This measure shows the fraction of the overall utility increase from a marginal increase in $y$ that is due to increased relative performance. The degree of positionality is zero if only absolute performance matters $\left(\beta^{i}>0, \zeta^{i}=0\right)$ and one if only relative performance matters $\left(\beta^{i}=0, \zeta^{i}>0\right)$. When both absolute and relative performance matter $\left(\beta^{i}>0, \zeta^{i}>0\right)$, the degree of positionality takes a value between zero and one.

If the agent fails in her attempt to perform the activity of her choosing, the realized value of $y$ is zero. In this situation, the pride function takes the value zero while the identity function takes the value $I^{i, f}=I\left(0-\bar{y}^{i}\right)<0$ where the super-index " $f$ " stands for failure. Since the agent nevertheless has spent time preparing for, and attempting to perform the activity, the time cost $h\left(y^{i}\right)$ is still present. ${ }^{6}$ The realized overall utility in the case of failure then becomes ${ }^{7}$ :

$$
U^{i, f}=u(w)+v\left(1-h\left(y^{i}\right)\right)+\zeta^{i} I\left(-\bar{y}^{i}\right) .
$$

The agent chooses $y^{i}$ to maximize the expected utility $\tilde{U}^{i}=p^{i} U^{i}+\left(1-p^{i}\right) U^{i f}$. Substituting the definitions of $U^{i}, U^{i f f}$ and $p^{i}$ into $\tilde{U}^{i}$, and maximizing the resulting expression w.r.t. $y^{i}$ produces the first-order condition:

$$
\tilde{U}_{y}^{i}=p^{i}\left(\beta_{E M B}^{i} g_{y}^{i}+\zeta^{i} I_{y}^{i}\right)+p_{y}^{i}\left[\beta^{i} g^{i}+\zeta^{i}\left(I^{i}-I^{i, f}\right)\right]-v_{z}^{i} h_{y}^{i}=0 .
$$

Equation (4) shows the trade-off the agent faces when choosing $y$. At the optimum, the expected marginal benefit $(E M B)$ of attempting to perform a more difficult activity is balanced against the marginal utility cost $(M U C)$ associated with

\footnotetext{
${ }_{5}^{5}$ In models where an agent's preferences are defined over private consumption, $\$ \$ c \$$, and relative consumption, $\$ \$$ Delta $=c-l s t a c k r e l\{-\}\{c\} \$ \$$, so that the utility function is given by $\$ \$ U \backslash l e f t(c, \mid$ Delta \right } \$ \$ \text { , an agent's degree of consumption positionality is defined as } \$ \$ \text { lalpha } = \{ U _ { - } \} _ { - } \{ \text { Delta } \} \wedge $\operatorname{left}\left(\{\mathrm{U}\}_{-}\{\mathrm{c}\}+\{\mathrm{U}\}_{-}\{\right.$Delta $\}$right $) \$$ (see e.g. Aronsson and Johansson-Stenman (2010)). The definition in Eq. (2) is analogous with this definition.

${ }^{6}$ One could also incorporate a direct utility cost associated with failure if failure is associated with injury. Including such a cost would not affect the qualitative results derived below.

7 It is also conceivable that attempting to climb a difficult mountain but failing may provide the agent with some satisfaction in the sense that "at least I tried". If this is the case then the sense of pride would still be there albeit not as strong as in the case of success. One way to model this feature would be to write the pride function in the case of failure as $g(k y)$, where $k$ is a positive scaling factor which is situated in the interval $0 \leq k<1$. If $k=0$, then $g(0)=0$ (as we have it in the text) but if $k$ is nonzero, then $0<g(k y)<g(y)$. If the "at least I tried" argument would also apply to the identity function, then the identity associated with failure would be modified to read $I(k y-\bar{y})$. If we were to use these alternative specifications then the agent would, all else equal, have an incentive to choose a larger $y$ than otherwise.
} 
choosing a more difficult alternative plus the marginal utility loss associated with giving up pure leisure time $\left(-v_{z}^{i} h_{y}^{i}\right)$. The $M U C$ reflects that attempting to perform a more risky activity, on the margin, reduces the probability of success, where failure implies a utility loss which is given by $U^{i}-U^{i, f}=\beta^{i} g^{i}+\zeta^{i}\left(I^{i}-I^{i, f}\right)$. Let $y^{i, \circ}$ denote the optimal choice which satisfies Eq. (4). This first-order condition implicitly defines $y^{i, \circ}$ as a function of $\beta^{i}, \zeta^{i}$ and $\bar{y}^{i}$, i.e. $y^{i, \circ}\left(\beta^{i}, \zeta^{i}, \bar{y}^{i}\right)$. In the Appendix, we show that $y^{i, \circ}>0$ as long as $\beta^{i}>0$. As for the second-order derivative, it is given by:

$$
\tilde{U}_{y y}^{i}=p^{i}\left(\beta^{i} g_{y y}^{i}+\zeta^{i} I_{y y}^{i}\right)+2 p_{y}^{i}\left(\beta^{i} g_{y}^{i}+\zeta^{i} I_{y}^{i}\right)+p_{y y}^{i}\left[\beta^{i} g^{i}+\zeta^{i}\left(I^{i}-I^{i f f}\right)\right]+v_{z z}^{i}\left(h_{y}^{i}\right)^{2}-v_{z}^{i} h_{y y}^{i} .
$$

Given the assumptions made above, it follows that the second-order condition $\tilde{U}_{y y}^{i}<0$ is satisfied which implies that the optimum is a global maximum. It can also be shown that if the two agent types are identical in all aspects except in the probability of success (i.e. $\beta^{1}=\beta^{2}, \zeta^{1}=\zeta^{2}$ and $\bar{y}^{1}=\bar{y}^{2}$ ) then $y^{2, \circ}>y^{1, \circ}$, i.e. the highskilled agent will choose to perform a more difficult activity than the low-skilled agent.

\section{Behavioral effects}

Let us now evaluate how an individual agent's optimal activity choice is affected by changes in $\bar{y}^{i}$ and $\zeta^{i}$. A change in $\bar{y}^{i}$ may reflect a changed behavior within the reference group while $\zeta^{i}$ may be interpreted as a taste shock whereby the agent attaches more importance to being a valued member of the social group. We begin with $\bar{y}^{i}$. Differentiating Eq. (4) w.r.t. $\bar{y}^{i}$ and $y^{i, \circ}$ produces:

$$
\frac{\partial y^{i, \circ}}{\partial \bar{y}^{i}}=-\frac{p^{i} \zeta^{i} I_{y \bar{y}}^{i}}{\tilde{U}_{y y}^{i}}-\frac{p_{y}^{i} \zeta^{i}\left(I_{\bar{y}}^{i}-I_{\bar{y}}^{i, f}\right)}{\tilde{U}_{y y}^{i}} .
$$

As a point of reference, consider first the outcome in the absence of uncertainty. In this situation $p^{i}=1$ and $p_{y}^{i}=0$ for all $y^{i} \geq 0$, in which case Eq. (6) reduces to $\partial y^{i, \circ} / \partial \bar{y}^{i}=-\zeta^{i} I_{y \bar{y}}^{i} / U_{y y}^{i}>0$, where $U_{y y}^{i}=\beta^{i} g_{y y}^{i}+\zeta^{i} I_{y y}^{i}+v_{z z}^{i}\left(h_{y}^{i}\right)^{2}-v_{z}^{i} h_{y y}^{i}<0$ and where we recall that $I_{y \bar{y}}^{i}>0$. This comparative static result reflects that an increase in $\bar{y}^{i}$, all else equal, has a negative impact on $\Delta^{i}=y^{i}-\bar{y}^{i}$ which, in turn, pushes up the marginal identity utility, $I_{y}^{i}$. When there is no risk of failure, the agent responds by choosing a higher $y^{i}$. This result is a consequence of the fact that the agent has keeping up with the Joneses preferences along the lines defined by Dupor and Liu (2003), i.e. that $U_{y \bar{y}}^{i}=\zeta^{i} I_{y \bar{y}}^{i}>0$. We therefore refer to this response as a conventional keeping up with the Joneses effect.

In the presence of uncertainty, the outcome is no longer clear-cut because the RHS of Eq. (6) is made up of two terms. The first $\left(-p^{i} \zeta^{i} I_{y \bar{y}}^{i} / \tilde{U}_{y y}^{i}>0\right)$ captures the 


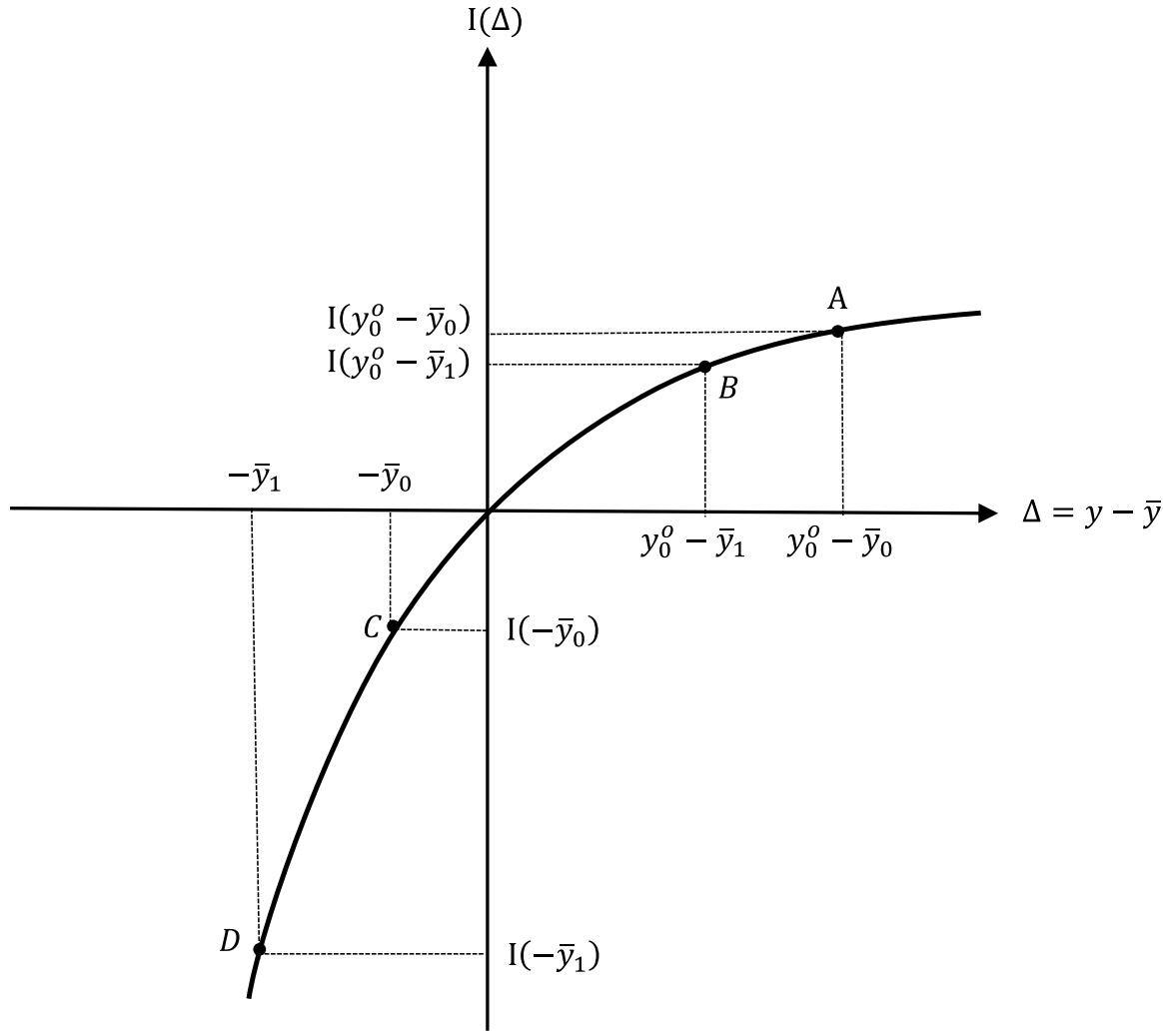

Fig. 1 Identity utility and relative performance

Joneses effect discussed above while the $\operatorname{second}^{8}\left(-p_{y}^{i} \zeta^{i}\left(I_{\bar{y}}^{i}-I_{\bar{y}}^{i, f}\right) / \tilde{U}_{y y}^{i}<0\right)$ reflects that an increase in the reference level magnifies the utility loss associated with failure. This potential utility loss provides the agent with an incentive to play it safe by choosing a lower $y^{i}$ than otherwise in order increase the probability of success. We will refer to this as the Cautionary effect of attaching more importance to an identity linked to a risky activity.

We illustrate the incentive underlying the Cautionary effect in Fig. 1 below where we, for notational convenience, omit the super-index $i$. Assume that the agent initially has made an optimal choice $y_{0}^{\circ}$ conditional on the observed initial reference level $\bar{y}_{0}$ (here the sub-index " 0 " refers to the initial value of a variable). The identity utility associated with success is therefore initially given by $I\left(y_{0}^{\circ}-\bar{y}_{0}\right)$ which corresponds to point $\mathrm{A}$ in Fig. 1 . If the reference level now increases to $\bar{y}_{1}>\bar{y}_{0}$ (where the sub-index " 1 " refers to the subsequent value of a

\footnotetext{
${ }^{8}$ From the concavity of the identity function, it follows that $I \frac{i}{y}-I \frac{i, f}{y}>0$ which makes it possible to sign the expression inside brackets.
} 
variable after a change has occurred), this affects the identity associated with success and moves the agent from point $\mathrm{A}$ to point $\mathrm{B}$. At point $\mathrm{B}$, the identity utility is given by $I\left(y_{0}^{\circ}-\bar{y}_{1}\right)$ before reoptimization takes place (i.e. before the agent has revised her initial choice $y_{0}^{\circ}$ ). The increase in the reference level also affects the identity associated with failure which is reduced from $I\left(-\bar{y}_{0}\right)$ at point $\mathrm{C}$, to $I\left(-\bar{y}_{1}\right)$ at point $\mathrm{D}$. Let $(A-C)$ and $(B-D)$ denote the vertical distance between points $\mathrm{A}$ and $\mathrm{C}$, and points $\mathrm{B}$ and $\mathrm{D}$, respectively, in Fig. 1. Since the identity function is concave, it follows that:

$$
(B-D)-(A-C)=\left[I\left(y_{0}^{\circ}-\bar{y}_{1}\right)-I\left(-\bar{y}_{1}\right)\right]-\left[I\left(y_{0}^{\circ}-\bar{y}_{0}\right)-I\left(-\bar{y}_{0}\right)\right]>0 .
$$

This means that the identity loss associated with failure is amplified when the reference level increases which, ceteris paribus, increases the overall utility loss associated with failure; $U-U^{f}=\beta g+\zeta\left(I-I^{f}\right)$. This provides the agent with an incentive to play it safe by choosing a lower $y$ and is the rationale underlying the Cautionary effect.

Whether the Joneses effect dominates over the Cautionary effect depends on the properties of the identity and probability functions. Let us introduce the following short notations: (i) let $\eta^{i}=-y^{i} I_{y \bar{y}}^{i} / I_{\bar{y}}^{i}>0$ be a measure of how sensitive the slope of the identity function is to a change in the reference level, (ii) let $\rho^{i}=-\left(I_{\bar{y}}^{i}-I_{\bar{y}}^{i, f}\right) / I_{\bar{y}}^{i}>0$ measure the relative difference in the slope of the identity function between the point of success and the point of failure and (iii) let $\varepsilon^{i, p}=-y^{i} p_{y}^{i} / p^{i}>0$ be the elasticity of the probability function w.r.t. $y^{i}$. By using these definitions, we can rewrite Eq. (6) to read

$$
\frac{\partial y^{i, \circ}}{\partial \bar{y}^{i}}=-\lambda^{i} \zeta^{i} I_{\bar{y}}^{i}\left(\eta^{i}-\rho^{i} \varepsilon^{i, p}\right),
$$

where $\lambda^{i}=-p^{i} / y^{i} \tilde{U}_{y y}^{i}>0$. Since $-\lambda^{i} \zeta^{i} I_{\bar{y}}^{i}>0$, we obtain the following results from (8):

Proposition 1: Consider an increase in the reference level, $\bar{y}^{i}$. The effect on agent type i's optimal choice $y^{i, \circ}$ can be summarized as follows:

(i) If $\eta^{i}>\rho^{i} \varepsilon^{i, p}\left(\eta^{i}<\rho^{i} \varepsilon^{i, p}\right)$ holds at the optimum,then the Joneses effect dominates over (is dominated by) the Cautionary effect, in which case $\partial y^{i, \circ} / \partial \bar{y}^{i}>0$ $\left(\partial y^{i, \circ} / \partial \bar{y}^{i}<0\right)$.

(ii) If $\eta^{i}=\rho^{i} \varepsilon^{i, p}$ holds at the optimum, then the Joneses effect and the Cautionary effect cancel out, in which case $\partial y^{i, \circ} / \partial \bar{y}^{i}=0$.

In the Appendix, we use specific functional forms to exemplify when $\eta^{i}-\rho^{i} \varepsilon^{i, p}$ is positive/negative at the optimum.

Let us now turn to the effects of an increase in the importance attached to being a valued member of the social group, i.e. an increase in the parameter $\zeta^{i}$. Differentiating Eq. (4) w.r.t. $\zeta^{i}$ and $y^{i, \circ}$ produces: 


$$
\frac{\partial y^{i, \circ}}{\partial \zeta^{i}}=-\frac{p^{i} I_{y}^{i}}{\tilde{U}_{y y}^{i}}-\frac{p_{y}^{i}\left(I^{i}-I^{i, f}\right)}{\tilde{U}_{y y}^{i}} .
$$

Also here we begin by noting that in the absence of uncertainty, Eq. (9) reduces to $\partial y^{i, \circ} / \partial \zeta^{i}=-I_{y}^{i} / U_{y y}^{i}>0$. This response reflects that when the importance attached to the identity utility increases, then the marginal benefit of choosing a higher $y^{i}$ increases which induces the agent to choose a larger $y^{i, \circ}$. Although this effect is not directly related to the sign of the cross-derivative $U_{y \bar{y}}^{i}=\zeta^{i} I_{y \bar{y}}^{i}$, we will nevertheless refer to this response as a keeping up with the Joneses effect.

In the presence of uncertainty, the comparative static effect in (9) is made up of two components; $-p^{i} I_{y}^{i} / \tilde{U}_{y y}^{i}>0$ which is the Joneses effect and $-p_{y}^{i}\left(I^{i}-I^{i, f}\right) / \tilde{U}_{y y}^{i}<0$ which reflects that if the agent attaches more importance than before to her identity, then the utility loss associated with failure will be magnified. ${ }^{9}$ This potential utility loss provides the agent with an incentive to play it safe. As such, this is a Cautionary effect associated with attaching more importance to the personal identity.

Let us define $\varepsilon^{i, I}=I_{y}^{i} y^{i} /\left(I^{i}-I^{i, f}\right)>0$ to be the elasticity of the identity rent of success w.r.t. $y^{i}$, where we use that $I_{y}^{i}=\partial\left(I^{i}-I^{i, f}\right) / \partial y^{i}$. By using the definition of $\varepsilon^{i, I}$ together with the definitions of $\varepsilon^{i, p}$ and $\lambda^{i}$ made above, we can rewrite Eq. (9) to read:

$$
\frac{\partial y^{i, \circ}}{\partial \zeta^{i}}=\left(\varepsilon^{i, I}-\varepsilon^{i, p}\right)\left(I^{i}-I^{i, f}\right) \lambda^{i} .
$$

We can now use this equation to evaluate when Joneses effect dominates over/is dominated by the Cautionary effect. The results can be summarized as follows:

Proposition 2: Consider an increase in the importance, $\zeta^{i}$, that an agent of type $i$ attaches to her identity. The effect on the optimal choice $y^{i, \circ}$ can be summarized as follows:

(i) If $\varepsilon^{i, I}>\varepsilon^{i, p}\left(\varepsilon^{i, I}<\varepsilon^{i, p}\right)$ holds at the optimum, then the Joneses effect dominates over (is dominated by) the Cautionary effect, in which case $\partial y^{i, \circ} / \partial \zeta^{i}>0$ $\left(\partial y^{i, \circ} / \partial \zeta^{i}<0\right)$.

(ii) If $\varepsilon^{i, I}=\varepsilon^{i, p}$ holds at the optimum, then the Joneses and the Cautionary effects cancel out, in which case $\partial y^{i, \circ} / \partial \zeta^{i}=0$.

In the Appendix, we use specific functional forms to exemplify when $\varepsilon^{i, I}-\varepsilon^{i, p}$ is positive/negative at the optimum.

\footnotetext{
${ }^{9}$ Since $U^{i}-U^{i, f}=\beta^{i} g^{i}+\zeta^{i}\left(I^{i}-I^{i f}\right)$, it follows that an increase in $\zeta^{i}$, all else equal, magnifies the utility difference between success and failure.
} 
An interesting special case arises if $\beta^{i}=0$, in which case the degree of positionality takes the value one ${ }^{10}$ :

Corollary: If only relative performance matters $\left(\alpha^{i}=1\right)$, then the first-order condition can be satisfied only if $\varepsilon^{i, I}>\varepsilon^{i, p}$ holds at the optimum. Then $\partial y^{i, \circ} / \partial \zeta^{i}>0$.

To show this result, note that when $\beta^{i}=0$, the first-order condition in (4) reduces to:

$$
0=p^{i} \zeta^{i} I_{y}^{i}+p_{y}^{i} \zeta^{i}\left(I^{i}-I^{i, f}\right)-v_{z}^{i} h_{y}^{i} \Rightarrow\left(\varepsilon^{i, I}-\varepsilon^{i, P}\right) \frac{\zeta^{i}\left(I^{i}-I^{i, f}\right) p^{i}}{y^{i}}=v_{z}^{i} h_{y}^{i}>0
$$

where we have used the definitions of $\varepsilon^{i, I}$ and $\varepsilon^{i, p}$ to rewrite the first order condition on the form presented in the second row. From the latter expression, it follows that the first-order condition can only be satisfied if $\varepsilon^{i, I}>\varepsilon^{i, P}$ holds at the optimum, in which case we know from part (i) in Proposition 2 that $\partial y^{i, \circ} / \partial \zeta^{i}>0$.

\section{The myopic equilibrium vs the first-best outcome}

A common result in the literature on positional preferences is that the incentive to keep up with the Joneses compels agents to consume more than they otherwise would have done which has a negative impact on welfare. The question we pose in this part is to what extent the over-consumption result holds when the positional activity has an uncertain outcome? To address this question, we compare the equilibrium with myopic agents with a first-best outcome.

Let us begin with the myopic equilibrium (ME). Recall that agent type $i$ 's demand function $y^{i, \circ}\left(\beta^{i}, \zeta^{i}, \bar{y}^{i}\right)$ is implicitly determined by the first-order condition in Eq. (4). For convenience, we state the first-order conditions for the two agent types here:

$$
\begin{aligned}
\tilde{U}_{y}^{1}= & p^{1}\left(y^{1, \circ}\right) \cdot\left[\beta^{1} \cdot g_{y}\left(y^{1, \circ}\right)+\zeta^{1} \cdot I_{y}\left(y^{1, \circ}-\bar{y}^{1}\right)\right]-v_{z}\left(1-h\left(y^{1, \circ}\right)\right) \cdot \\
& h_{y}\left(y^{1, \circ}\right)+p_{y}^{1}\left(y^{1, \circ}\right) \cdot\left\{\beta^{1} \cdot g\left(y^{1, \circ}\right)+\zeta^{1} \cdot\left[I\left(y^{1, \circ}-\bar{y}^{1}\right)-I^{f}\left(-\bar{y}^{1}\right)\right]\right\}=0 . \\
\tilde{U}_{y}^{2}= & p^{2}\left(y^{2, \circ}\right) \cdot\left[\beta^{2} \cdot g_{y}\left(y^{2, \circ}\right)+\zeta^{2} \bullet I_{y}\left(y^{2, \circ}-\bar{y}^{2}\right)\right]-v_{z}\left(1-h\left(y^{2, \circ}\right)\right) \cdot \\
& h_{y}\left(y^{2, \circ}\right)+p_{y}^{2}\left(y^{2, \circ}\right) \cdot\left\{\beta^{2} \cdot g\left(y^{2, \circ}\right)+\zeta^{2} \cdot\left[I\left(y^{2, \circ}-\bar{y}^{2}\right)-I^{f}\left(-\bar{y}^{2}\right)\right]\right\}=0 .
\end{aligned}
$$

${ }^{10}$ We are thankful to an anonymous referee for making us aware of this possibility. 


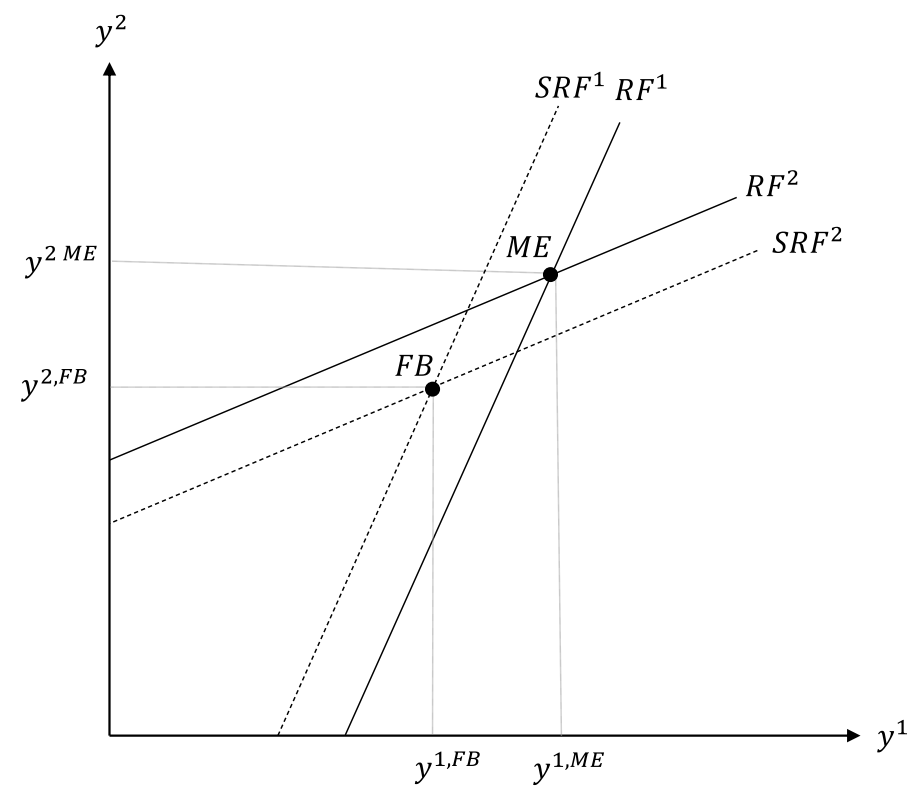

Fig. 2 Myopic equilibrium (ME) and First Best (FB) solution under Joneses dominance

If we substitute $\bar{y}^{1}=x^{1} y^{1, \circ}+\left(1-x^{1}\right) y^{2}$ into (12), the resulting equation implicitly defines $y^{1, \circ}$ as a function of $y^{2}$, as well as a function of $\beta^{1}, \zeta^{1}$ and $x^{1}$. Let us denote this reaction function $R F^{1}=y_{R F}^{1, \circ}\left(y^{2}, \beta^{1}, \zeta^{1}, x^{1}\right)$. The other agent type's reaction function is denoted $R F^{2}=y_{R F}^{2, \circ}\left(y^{1}, \beta^{2}, \zeta^{2}, x^{2}\right)$ and it is analogously defined by substituting $\bar{y}^{2}=x^{2} y^{1}+\left(1-x^{2}\right) y^{2, \circ}$ into Eq. (13). Differentiating $R F^{1}$ and $R F^{2}$ produces the following comparative static results (slopes) ${ }^{11}$ :

$$
\frac{\partial y_{R F}^{1, \circ}}{\partial y^{2}}=\frac{1-x^{1}}{1-x^{1} \frac{\partial y^{1, \circ}}{\partial \bar{y}^{1}}} \frac{\partial y^{1, \circ}}{\partial \bar{y}^{1}}, \frac{\partial y_{R F}^{2, \circ}}{\partial y^{1}}=\frac{1-x^{2}}{1-x^{2} \frac{\partial y^{2, \circ}}{\partial \bar{y}^{2}}} \frac{\partial y^{2, \circ}}{\partial \bar{y}^{2}}
$$

From these equations it follows that as long a $x^{i} \partial y^{i, \circ} / \partial \bar{y}^{i}<1$, then the slope of agent type $i$ 's reaction function is determined by the sign of the partial derivative $\partial y^{i, \circ} / \partial \bar{y}^{i}$. Since we know from Proposition 1 that the sign of $\partial y^{i, \circ} / \partial \bar{y}^{i}$ depends on whether or not the Joneses effect dominates over the Cautionary effect, it follows that also the slope of agent type $i$ 's reaction function depends on these effects. The myopic equilibrium is at the point where the two reaction functions intersect, and the equilibrium levels are denoted $y^{1, M E}$ and $y^{2, M E}$. Figure 2 below illustrates the outcome when the Joneses effect dominates over the Cautionary effect for both agent types (i.e. $\partial y^{i, \circ} / \partial \bar{y}^{i}>0$ holds for $i=1,2$ ), and the two reaction functions have positive slopes. In Fig. 2, we see that the two reaction functions will intersect, and the

11 See the Appendix. 
(a) $y^{2}$

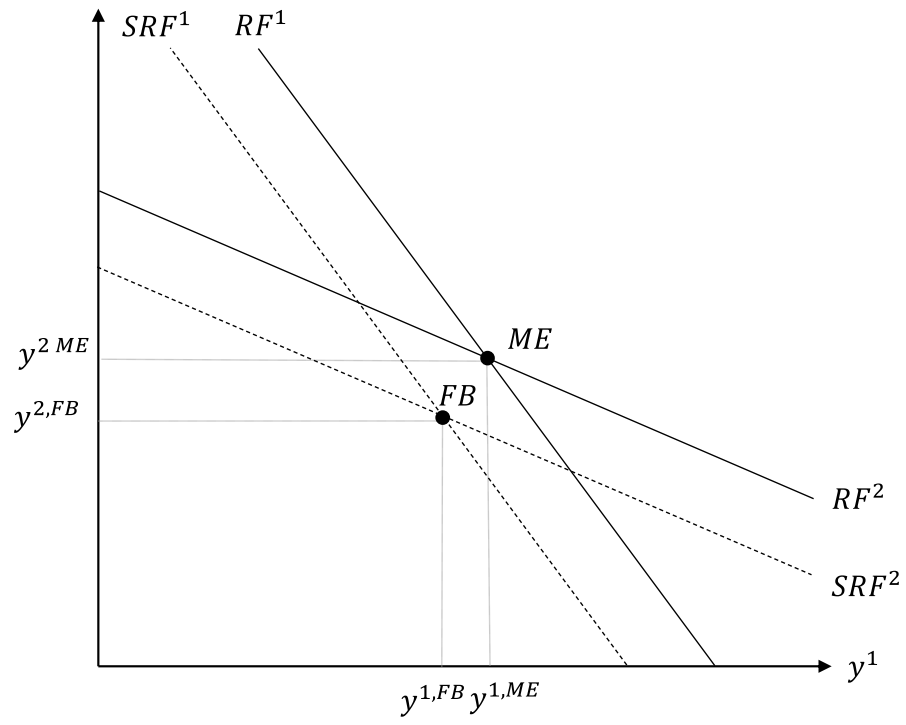

(b) $y^{2}$

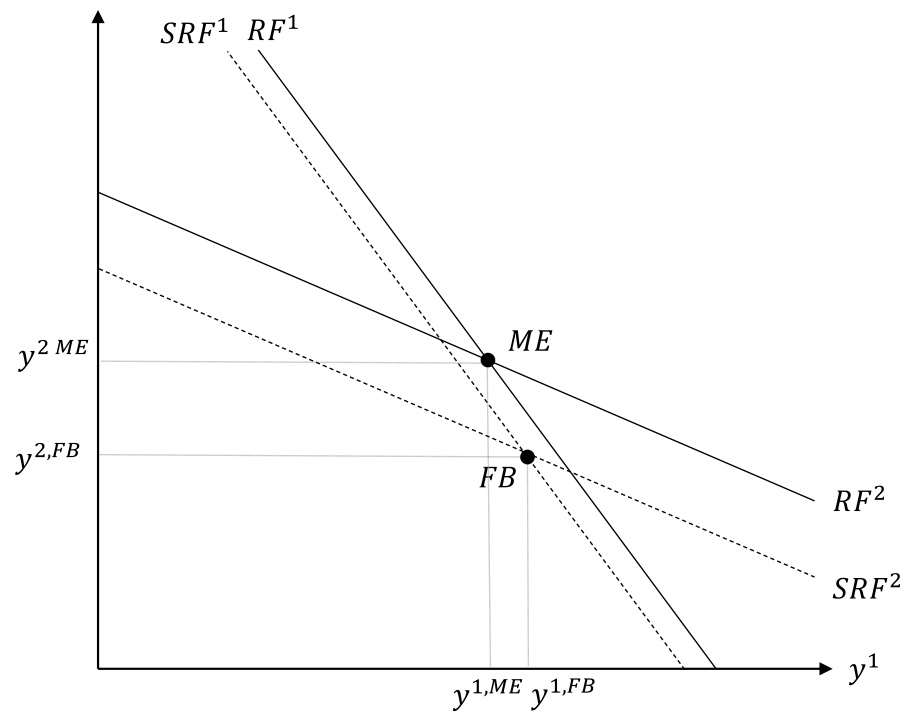

Fig. 3 a ME and FB solution with over-consumption for both agent types under Cautionary dominance. b ME and FB solution with both over- and under-consumption under Cautionary dominance 
myopic equilibrium will exist, if $R F^{2}$ has a flatter slope in $\left(y^{1}, y^{2}\right)$ space than $R F^{1}$. In the Appendix, we show that this happens if $\partial y^{i, \circ} / \partial \bar{y}^{i}<1$ for $i=1,2{ }^{12}$ Figures $3 \mathrm{a}$, $\mathrm{b}$ below illustrate two possible outcomes when instead it is the Cautionary effect which dominates over the Joneses effect for both agent types (i.e. $\partial y^{i, \circ} / \partial \bar{y}^{i}<0$ for $i=1,2$ ), in which case the two reaction functions are negatively sloped.

Let us now turn to the first-best (FB) outcome. It is obtained by considering a social planner who maximizes the expected welfare within the social group. The expected utilitarian welfare function is given by $\tilde{W}=N^{1} \tilde{U}^{1}+N^{2} \tilde{U}^{2}$, where $N^{i}$ is the number of agents of type $i$ within the social group. The social planner chooses $y^{1}$ and $y^{2}$ to maximize $\tilde{W}$, while taking into account how these choices affect the reference levels $\bar{y}^{1}=x^{1} y^{1}+\left(1-x^{1}\right) y^{2}$ and $\bar{y}^{2}=x^{2} y^{1}+\left(1-x^{2}\right) y^{2}$. Substituting the latter two equations into the welfare function and maximizing w.r.t. $y^{1}$ and $y^{2}$ produces the following necessary conditions associated with the first-best optimum:

$$
\begin{gathered}
\frac{\partial \tilde{W}}{\partial y^{1}} \frac{1}{N^{1}}=\tilde{U}_{y}^{1}+\frac{1}{N^{1}} \sum_{i} N^{i} x^{i} \tilde{U}_{\bar{y}}^{i}=0, \\
\frac{\partial \tilde{W}}{\partial y^{2}} \frac{1}{N^{2}}=\tilde{U}_{y}^{2}+\frac{1}{N^{2}} \sum_{i} N^{i}\left(1-x^{i}\right) \tilde{U}_{\bar{y}}^{i}=0,
\end{gathered}
$$

where $\tilde{U}_{\bar{y}}^{i}=p^{i} \zeta^{i} I_{\bar{y}}^{i}+\left(1-p^{i}\right) \zeta^{i} I_{\bar{y}}^{i, f}<0$. To depict the first-best outcome $\left(y^{1, F B}, y^{2, F B}\right)$, we can use that Eq. (15) implicitly defines $y^{1}$ as a function of $y^{2}$ while Eq. (16) implicitly defines $y^{2}$ as a function of $y^{1}$. Let us refer to these functional relationships as social reaction functions, and denote them $S R F^{1}$ and $S R F^{2}$, respectively. Since the social planner accounts for the fact that $y^{1}$ and $y^{2}$ produce negative externalities on all agents in the social group via the reference levels $\bar{y}_{1}$ and $\bar{y}_{2}$, while this is not taken into account by the myopic private agents, it follows that $S R F^{2}$ will be situated below $R F^{2}$ in $\left(y^{1}, y^{2}\right)$ space while $S R F^{1}$ will be situated to the left of $R F^{1}$ in $\left(y^{1}, y^{2}\right)$ space. The social reaction functions are depicted in Figs. 2, 3a, b below, and the first-best outcome is at the point where $S R F^{1}$ and $S R F^{2}$ intersect.

Figure 2 illustrates the outcome when the Joneses effect dominates over the Cautionary effect. In this situation, there is over-consumption in the myopic equilibrium in the sense that $y^{i, F B}<y^{i, M E}$ holds for $i=1,2$. This over-consumption result is analogous to results derived in earlier literature on positional preferences, where it has been shown that inter-personal comparisons induce agents to engage in wasteful competition, thereby inducing agents to over-consume positional goods.

If, instead, the Cautionary effect dominates over the Joneses effect, the outcome is no longer clear-cut. Figure 3a illustrates an outcome where both agent types overconsume the acticity while Fig. $3 b$ illustrates an outcome where one of the agent types, type 1 in this example, under-consumes the activity.

Let us take a closer look on the case where there is under-consumption among one of the agent types. To do this, let us consider the empirically interesting case

$\overline{{ }^{12} \text { Note that if } \partial y^{i, o} / \partial \bar{y}^{i}<1 \text { holds }}$ for $i=1,2$, then it follows that also $x^{i} \partial y^{i, \circ} / \partial \bar{y}^{i}<1$ is satisfied. 


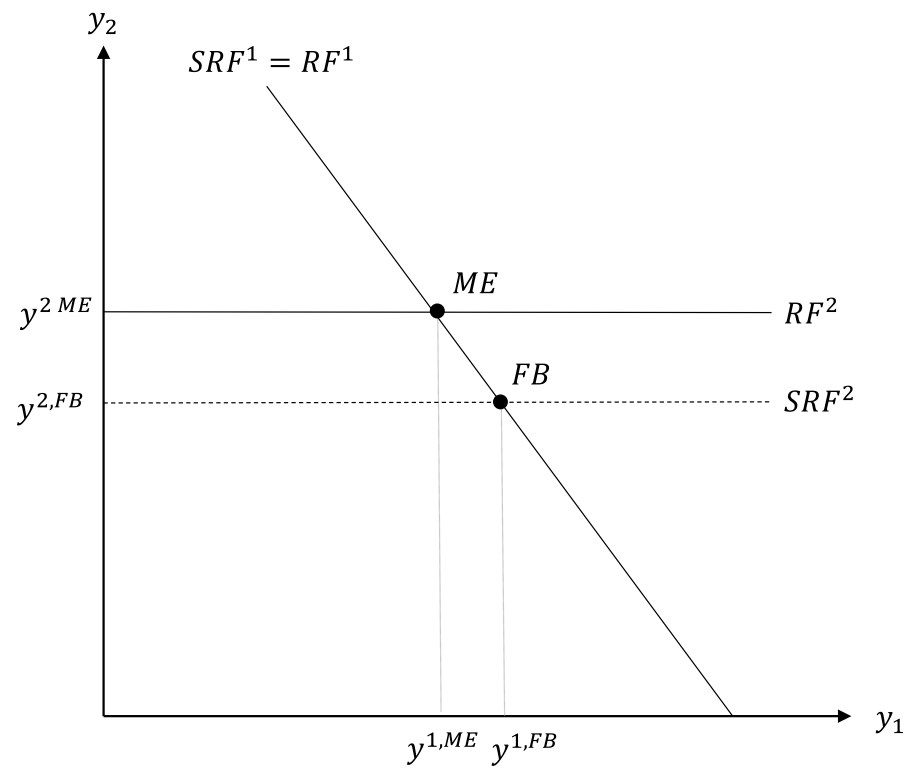

Fig. $4 \mathrm{ME}$ and FB under Cautionary dominance and elitist social preferences

where all members in the social group only compare themselves with the elite, which is the group of high-skilled agents of type 2 (see e.g., Cowan 2004; Clark and Senik 2010; Eckerstorfer and Wendner 2013). This implies $x^{1}=x^{2}=0$ and $\bar{y}^{1}=\bar{y}^{2}=y^{2}$, in which case the low-skilled agents do not contribute to the positional externality and the social first-order condition for $y^{1}$ reduces to $\partial \tilde{W} / \partial y^{1}=\tilde{U}_{y}^{1}=0$. Since $\tilde{U}_{y}^{1}=0$ is the equation which implicitly defines the $R F^{1}$, it follows that $S R F^{1}$ coincides with $R F^{1}$ when $\bar{y}^{1}=\bar{y}^{2}=y^{2}$. Since $R F^{1}$ has a negative slope when the Cautionary effect dominates over the Joneses effect for agent type 1, it follows that also $S R F^{1}$ has a negative slope in this special case. As for the other social reaction function $S R F^{2}$, we note that $y^{1}$ does not appear in the social first-order condition for $y^{2}$ when $\bar{y}^{2}=y^{2}$. Therefore Eq. (16) defines the first-best level $y^{2, F B}$ independently of $y^{1}$. Hence, $S R F^{2}$ is a horizontal line which is situated below ${ }^{13} R F^{2}$ in $\left(y^{1}, y^{2}\right)$ space. The outcome is illustrated in Fig. 4 where $y^{1, F B}>y^{1, M E}$ and $y^{2, F B}<y^{2, M E}$. Hence, on a free market, positional preferences for risky activities may induce some agents to under-consume the leisure activity in the sense that the agent chooses a difficulty level which is too low from the perspective of the social planner. This result is consistent with research in social psychology, according to which individuals with a lower self-perceived status (e.g. due to a lower skill level) tend to avoid risk as a self-protective action (Baumeister et al. 1989; Wolfe et al. 1986).

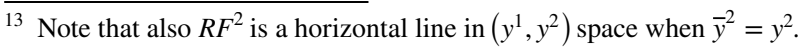


Since $\partial y_{R F}^{1, \circ} / \partial y_{2}=\partial y^{1, \circ} / \partial \bar{y}^{1}$ when $x_{1}=x_{2}=0$, and since we know from Proposition 1 that the sign of $\partial y^{1, \circ} / \partial \bar{y}^{1}$ is determined by the sign of $\eta^{1}-\rho^{1} \varepsilon^{1, p}$, we can combine these observations to obtain the following results:

Proposition 3: Consider a social group made up of two agent types $i=1,2$, where all agents have positional preferences w.r.t. a risky leisure activity and compare themselves with the high-skilled agents of type 2. Compared with the first-best outcome, the outcome in the myopic equilibrium has the following properties:

(i) In the myopic equilibrium, the high-skilled agent's activity choice exceeds the first-best choice, i.e. $y^{2, M E}<y^{2, F B}$ (over-consumption).

(ii) If $\eta^{1}<\rho^{1} \varepsilon^{1, p}$, the low-skilled agent's activity choice in the myopic equilibrium falls short of the first-best choice, i.e. $y^{1, M E}<y^{1, F B}$ (under-consumption).

(iii) If $\eta^{1}>\rho^{1} \varepsilon^{1, p}$, the low-skilled agent's activity choice in the myopic equilibrium exceeds the first-best choice, i.e. $y^{1, M E}>y^{1, F B}$ (over-consumption).

\section{Policy implications}

The positional externality has a detrimental effect on the welfare for all agents in the social group. From earlier studies, it is well known that public policy can be used (at least partially) to internalize the positional externality generated by interpersonal comparisons of income or consumption (e.g. Aronsson and Johansson-Stenman, 2008, 2010). In this part, we ask whether public policy also can play a role when the positional externality is related to a risky leisure activity? We focus on the case where all members in the social group compare themselves with high-skilled agents but it is straightforward to extend the results presented below into a more general framework.

We proceed along two routes. First, we ask what type of policy instrument a policymaker needs to have at her disposal in order to implement the first-best policy. In the conventional literature on positional consumption goods, policy-makers can use taxes on income and/or positional commodities to improve the welfare. Unfortunately, such tax instruments may not offer viable alternatives when agents have positional concerns for leisure activities. Instead, we will use the fact that some risky leisure activities take place on publicly owned land. If the areas where agents perform their activities (e.g. trails or rivers) can be assigned unique index values based on their respective difficulty levels, and if the policy-maker can implement and monitor payment of fees, then the policy-maker has the possibility to impose an entrance fee to access an activity with a given index level $y$. These fees can e.g. be entrance fees to national parks, trailfees for mountain bikers/hikers or participation fees for long-distance running races. In Sect. 5.1, we show that an optimal design of this entrance cost function makes it possible to implement the first-best outcome defined in Section IV. However, a drawback of this approach is that it requires the policy-maker to monitor the activities on all public land. In addition, it does not include activities on private land. Therefore, we 
in Sect. 5.2, instead base the optimal policy on an alternative approach; namely that if public assistance or a rescue effort is required to bring the agent back to safe grounds, then he/she should pay for this effort.

\subsection{First-best policy: an optimal entrance cost function}

Assume that the policy-maker is able to implement and monitor a system where the agents are required to pay a fee to perform an activity with index/difficulty level $y$. We allow the fee facing the agents to be a nonlinear function of the activity's difficulty index levels, $\varphi(y)$, and assume that the revenue generated by the entrance fees is used to cover some fixed expenditure $E$. These expenditures can, for example, be used on safety arrangements (e.g. shelters, emergency phones, maintainance of trails, etc.). The policy-maker's budget constraint therefore becomes $E=\sum_{i} N^{i} \varphi\left(y^{i}\right)$ and as mentioned above, we focus on the case where all agent compare themselves with the elite, i.e. $\bar{y}^{1}=\bar{y}^{2}=\bar{y}=y^{2}$.

The inclusion of an entrance cost implies that the private agent's budget constraint is modified to read $c^{i}=w-\varphi\left(y^{i}\right)$. By using this modified budget constraint in the private agent's decision problem, it is straightforward to show that the first-order condition for the optimal choice of $y^{i}$ in Eq. (4), now contains an additional term; $-\varphi_{y}^{i} u_{c}^{i}$. If the marginal entrance cost $\left(\varphi_{y}^{i}\right)$ facing an agent of type $i$ is positive, the additional term in the private first-order condition is negative and contributes to increase the marginal utility cost of attempting to perform a more difficult task. This modification of the model implies that in addition to being a function of $\beta^{i}, \zeta^{i}$ and $\bar{y}^{i}$, agent type $i^{\prime}$ s optimal choice $y^{i, \circ}$ now also becomes a function of $w$ and the parameters of the entrance cost function.

The policy-maker maximizes the social welfare function $\tilde{W}=\sum_{i} N^{i} \tilde{U}^{i}$ subject to the public budget constraint $E=\sum_{i} N^{i} \varphi\left(y^{i}\right)$. The decision variables are the parameters of the entrance cost function and the policy-maker recognizes the private time constraint in the maximization problem. It is also assumed that the policy-maker knows the proportion of each skill-type within the population (i.e. $N^{i} / \sum_{i} N^{i}$ is known) but the skill level of an individual agent may not be known by the policy-maker. This information asymmetry means that an agent of type $i$ has the possibility to choose to perform the task with difficulty level $y^{j, F B}$, where $j=1,2$ and $i \neq j$, instead of difficulty level $y^{i, F B}$ where the latter is the first-best choice intended for agent type $i$. To prevent this behavior, we need to impose an incentive compatibility (IC) constraint for each agent type. However, it can be shown that these IC constraints will not be binding for any of the agent types as long as the first-best policy features $y^{1, F B}<y^{2, F B}$ and this is the case that we will focus on. The Lagrange function associated with the policy-maker's maximization problem can therefore be written as follows:

$$
L=\sum_{i} N^{i} \tilde{U}^{i}+\gamma\left[\sum_{i} N^{i} \varphi\left(y^{i}\right)-E\right]+\mu\left(\bar{y}-y^{2}\right),
$$

where $\gamma$ and $\mu$ are Lagrange multipliers associated with the policy-maker's budget constraint and the reference level, respectively. By writing the reference level as an explicit Lagrange restriction, $\bar{y}$ will be treated as an additional (and artificial) 
decision-variable in the policy-maker's maximization problem. This approach makes it possible to define the quotient of Lagrange multipliers $\mu / \gamma$ as the shadow price (in real terms) of $\bar{y}$. This shadow price is interpretable as the marginal value that the policy-maker attaches to a reduction in the reference level, measured in terms of the revenue generated by the entrance fees. Before characterizing the optimal policy implied by the social first-order conditions, we recall that the impact of an increase in the reference level on agent type $i$ 's expected utility is determined by $\tilde{U}_{\bar{y}}^{i}=p^{i} \zeta^{i} I_{\bar{y}}^{i}+\left(1-p^{i}\right) \zeta^{i} I_{\bar{y}}^{i, f}<0$. We can therefore use this equation to define agent type $i$ 's marginal willingness to pay for a reduction in the reference level as:

$$
M W P_{\bar{y}, w}^{i}=-\frac{\tilde{U}_{\bar{y}}^{i}}{\tilde{U}_{w}^{i}}=-\frac{\zeta^{i} p^{i} I_{\bar{y}}^{i}+\zeta^{i}\left(1-p^{i}\right) I_{\bar{y}}^{i, f}}{u_{c}^{i}}>0,
$$

where we in the second step have used that $\tilde{U}_{w}^{i}=u_{c}^{i}$ at the optimum. In the Appendix, we show that the policy outlined in the following Proposition replicates the first-best outcome defined in Sect. 4;

Proposition 4: The following policy reproduces the first-best choices:

$$
\frac{\mu}{\gamma}=\sum_{i} N^{i} M W P_{\bar{y}, w}^{i}, \varphi_{y}^{1}=0, \varphi_{y}^{2}=\frac{\mu}{\gamma} \frac{1}{N^{2}}=\frac{\sum_{i} N^{i} M W P_{\bar{y}, w}^{i}}{N^{2}}
$$

The first equation in Proposition 4 shows that the shadow price is equal to the sum of the marginal willingness to pay for a reduction in the reference level. This shadow price constitutes the basis for constructing the optimal marginal entrance cost facing the high-skilled agent, which is determined by the third equation in Proposition 4. That equation shows that the positional externality is fully internalized when the marginal entrance cost facing the high-skilled agent is set equal to the sum of all agents' marginal willingness to pay for a reduction in the reference level, scaled by $N^{2}$. The scaling of the shadow price reflects that the individual highskilled agent only contributes a fraction $1 / N^{2}$ to the positional externality. Finally, the second equation in Proposition 4 shows that there is no motive to distort the lowskilled agent's choice as long as the marginal entrance cost facing the high-skilled agent fully internalizes the positional externality. Hence $\varphi_{y}^{1}=0$.

\subsection{Second-best policy: an optimal assistance cost function}

The monitoring cost of implementing the optimal entrance cost function is likely to be overwhelmingly high. An alternative is to introduce a fee that is activated when things go wrong. Many types of risky leisure activities, including mountaineering, hiking, backcountry skiing, mountain-biking etc. take place in relatively remote areas. A failure in these activities can involve getting lost, injured, or energy depleted. The remote location, and rough terrain, means that rescue operations are costly. In many western economies, taxes fund these rescue operations. Now, suppose that an agent who needs public assistance to get to safety, will have to pay 
a fee for the effort needed to carry out the operation. In this part, we analyze the optimal design of such an assistance cost function. To address this issue, we modify the model outlined above as follows. First, we make the assumption that if an agent of type $i$ fails to perform a task with difficulty level $y^{i}$, she needs assistance with probability $1-q^{i}\left(y^{i}\right)$, and does not need assistance with probability $q^{i}\left(y^{i}\right)$. We assume that $q^{i}\left(y^{i}\right)$ is type specific and decreasing in $y^{i}$. If assistance is needed, the agent pays a fee where the size of the fee depends on the difficulty level of the task; $\phi^{i}=\phi\left(y^{i}\right)$. As in the previous section, we allow the assistance cost function to be a function of $y^{i}$. If an agent succeeds with the activity or if she fails but does not need assistance, the private budget constraint is given by $c^{i}=w$. However, if the agent fails with the task and needs assistance, then the private budget constraint is modified to read $c^{i, f}=w-\phi\left(y^{i}\right)$. The utility associated with successfully preforming the activity $y^{i}$, is defined by Eq. (1) while the outcome of failure is now uncertain and depends on whether assistance is needed or not. The expected utility associated with failure, denoted $\tilde{U}^{i f f}$, is given by:

$$
\tilde{U}^{i, f}=q^{i} u(w)+\left(1-q^{i}\right) u\left(w-\phi\left(y^{i}\right)\right)+v\left(1-h\left(y^{i}\right)\right)+\zeta^{i} I(-\bar{y}),
$$

where $q^{i}=q^{i}\left(y^{i}\right)$. The agent chooses $y^{i}$ to maximize $\tilde{U}^{i}=p^{i} U^{i}+\left(1-p^{i}\right) \tilde{U}^{i, f}$ and the private first-order condition becomes:

$$
\begin{aligned}
\tilde{U}_{y}^{i}= & p^{i}\left(\beta^{i} g_{y}^{i}+\zeta^{i} I_{y}^{i}\right)+p_{y}^{i}\left[\beta^{i} g^{i}+\zeta^{i}\left(I^{i}-I^{i, f}\right)\right]-v_{z}^{i} h_{y}^{i} \\
& -\left(1-p^{i}\right)\left(1-q^{i}\right) u_{c}^{i, f} \phi_{y}^{i}+\left[p_{y}^{i}\left(1-q^{i}\right)+\left(1-p^{i}\right) q_{y}^{i}\right]\left(u-u^{i, f}\right)=0
\end{aligned}
$$

where $u=u(w)$ and $u^{i f}=u\left(w-\phi\left(y^{i}\right)\right)$. The terms in the first row are the same as those appearing in Eq. (4) while the terms in the second row are novel. The first term in the second row reflects that in the case of failure, there is now a risk of needing assistance which is associated with a cost. If the marginal assistance cost $\phi_{y}^{i}$ is positive, the first term in the second row contributes to increase the marginal utility cost of attempting to perform a more difficult activity. The second additional term, which is negative, reflects that attempting to perform a more difficult task is associated with a higher probability of needing assistance, where $u-u^{i, f}$ is the additional utility loss that is incurred if assistance is needed.

Turning to the policy-maker, we first note that the expected number of assistance missions provided to agents of type $i$ is given by $\tilde{N}^{i}\left(y^{i}\right)=\left[1-p^{i}\left(y^{i}\right)\right]\left[1-q^{i}\left(y^{i}\right)\right] N^{i}$. The cost of assisting an individual agent who has tried, but failed to perform $y^{i}$ is captured by a cost function $C\left(y^{i}\right)$ which is increasing and convex in $y^{i}$, i.e. $C_{y}^{i}=C_{y}\left(y^{i}\right)>0, C_{y y}^{i}=C_{y y}\left(y^{i}\right)>0$. Since each agent who receives assistance after having attempted task $y^{i}$ pays the fee $\phi\left(y^{i}\right)$, the budget constraint that the policymaker (in expectation) faces when she determines the parameters of the assistance cost function can be written as $E=\sum_{i} \tilde{N}^{i}\left(y^{i}\right)\left[\phi\left(y^{i}\right)-C\left(y^{i}\right)\right]$. The Lagrange function associated with the policy-maker's maximization problem is specified as follows: 


$$
L=\sum_{i} N^{i} \tilde{U}^{i}+\gamma\left[\sum_{i} \tilde{N}^{i}\left(y^{i}\right)\left[\phi\left(y^{i}\right)-C\left(y^{i}\right)\right]-E\right]+\mu\left(\bar{y}-y_{2}\right) .
$$

We solve this maximization problem in the Appendix. Before we present the results, define:

$$
\pi^{i}=\frac{\tilde{U}_{w}^{i}}{u_{w}^{i, f}}=p^{i} \frac{u_{w}}{u_{w}^{i, f}}+\left(1-p^{i}\right) q^{i} \frac{u_{w}}{u_{w}^{i, f}}+\left(1-p^{i}\right)\left(1-q^{i}\right)<1,
$$

where we have used that $u_{w}^{i, f}=u_{w}^{f}\left(w-\phi\left(y^{i}\right)\right)>u_{w}(w)$ to obtain the inequality on the RHS in (21). After these preliminaries, we are able to summarize the optimal second-best policy as follows:

Proposition 5: The optimal design of the assistance cost function has the following characteristics:

$$
\frac{\mu}{\gamma}=\sum_{i} \pi^{i} N^{i} M W P_{\bar{y}, w}^{i}, \phi_{y}^{1}=C_{y}^{1}-\frac{\left(\phi^{1}-C^{1}\right)}{\tilde{N}^{1}} \tilde{N}_{y}^{1}, \phi_{y}^{2}=C_{y}^{2}-\frac{\left(\phi^{2}-C^{2}\right)}{\tilde{N}^{2}} \tilde{N}_{y}^{2}+\frac{1}{\tilde{N}^{2}} \frac{\mu}{\gamma} .
$$

Since $\pi^{i}<1$, the first equation in Proposition 5 implies that the shadow price in the second-best optimum is not equal to the sum of the marginal willingness to pay for a reduction in the reference level. Instead $\mu / \gamma<\sum_{i} N^{i} M W P_{\bar{y}, w}^{i}$. To explain this result, we recall that the shadow price reflects the marginal value that the policymaker attaches to a reduction in the reference level, measured in terms of the revenue generated by the assistance fees. At the optimum, the optimal second-best policy will feature the equalization of the social marginal value of raising one extra dollar vis-à-vis the private marginal utility cost of giving up one extra dollar. Since the policy-maker is restricted to raise revenue from agents who need assistance, the private marginal utility cost of giving up one extra dollar will be given by $u_{w}^{i, f}=u_{w}^{f}\left(w-\phi\left(y^{i}\right)\right)$. Hence, the optimal second-best policy features $\gamma=u_{w}^{i, f}$ for $i=1,2$ and since the agents who do not need assistance do not pay any fee, it follows that $u_{w}(w)<u_{w}^{i, f}$ for $i=1,2$. The inability to distribute the cost of assistance equally between the two relevant states of nature (assistance needed or not needed) for an agent of type $i$ means that the social cost of raising one extra dollar $(\gamma)$ will be larger in the second-best than in the first-best. As a consequence, the shadow price will be smaller in the second-best than in the first-best, where we recall that the shadow price in the first-best equals the sum of the marginal willingness to pay for a reduction in the reference level. This explains why $\mu / \gamma<\sum_{i} N^{i} M W P_{\bar{y}, w}^{i}$ in the second-best optimum.

Let us now turn to the optimal marginal assistance cost facing the low-skilled agent. The second equation in Proposition 5 shows that the marginal assistance cost facing the low-skilled agent, $\phi_{y}^{1}$, is made up of two parts. The first part, $C_{y}^{1}$, reflects that the marginal assistance fee should be equal to the actual marginal assistance 
cost at the optimum. By setting $\phi_{y}^{1}$ equal to $C_{y}^{1}$, the low-skilled agent is provided with the correct incentive when it comes to internalizing the cost associated with assisting the agent. The second term in the formula for $\phi_{y}^{1}$ captures a revenue effect; if the low-skilled agent's net revenue contribution is positive (negative), such that $\phi^{1}>C^{1}\left(\phi^{1}<C^{1}\right)$, then the policy-maker has an incentive to increase (decrease) the tax base, i.e. increase (decrease) $\tilde{N}^{1}\left(y^{1}\right)$, by implementing a smaller (larger) marginal assistance fee than otherwise. ${ }^{14}$ Note that neither of these terms appeared in the corresponding formula defined in the previous section for the marginal entrance cost facing the low-skilled agent. ${ }^{15}$

The first two terms in the formula for the marginal assistance cost facing the high-skilled agent, $\phi_{y}^{2}$, that is presented in the third equation in Proposition 5 are similar to, and can be interpreted in a similar way, as the terms that appear in the formula for $\phi_{y}^{1}$. The third term in the expression for $\phi_{y}^{2}$ serves the purpose of (partly) internalizing the positional externality. As in Sect. 5.1, the shadow price constitutes the basis for constructing the corrective part of the marginal assistance cost facing the high-skilled agent. Note, however, that the scaling differs compared with that which appears in the first-best. In the first-best, the shadow price is scaled by $N^{2}$ whereas the shadow price is scaled by $\tilde{N}^{2}$ in the second-best. The explanation for why the shadow price is scaled by $\tilde{N}_{2}$ in the second-best is that it is not certain that a high-skilled agent will need assistance. Therefore, the corrective part of the marginal assistance cost needs to be higher than otherwise in order to induce the highskilled agent to choose a level of $y^{2}$ closer to the first-best. However, since the private marginal utility cost of giving up one extra dollar is higher in the second-best than in the first-best (see the discussion following Proposition 5), the corrective part of the marginal assistance fee will nevertheless not be sufficient to implement the first-best outcome.

\section{Concluding discussion}

In this paper, we have developed a model where agents have positional preferences for a risky leisure activity, which holds a social value. Our theoretical analysis suggests that positional preferences can both increase and decrease risk-taking behavior. This result is in stark contrast to those derived under certainty where positional preferences always increases investments in the positional activity. We find that the elasticity of the probability of success w.r.t. the positional activity determines how the agent responds. If changes in the probability of success have a small impact on behavior, then positional preferences increases engagement in the risky activity. For sufficiently large values of the elasticity of the probability of success, however, the

\footnotetext{
${ }^{14}$ This effect is analogous to tax base effects that appear in second-best tax formulas in the optimal tax literature.

${ }^{15}$ Since the system defined in Sect. 5.1 is based on entrance fees instead of assistance fees, it follows that $\$ \$\{C\}_{-}\{y\}^{\wedge}\{1\} \$ \$$ is redundant in the former system. Furthermore, since the entrance fee defined in Sect. 5.1 is paid by the agent regardless of whether she succeeds or fails, the tax base for the low-skilled agents is fixed at $\$ \$\{N\}^{\wedge}\{1\} \$ \$$. This means that tax base effects are redundant in that setting.
} 
agent instead responds by reducing her level of risk exposure (i.e. the agent responds by attempting to perform a less difficult task). The latter result has implications for the social equilibrium. To illustrate the effect, we focus on the empirically relevant case where all individuals compare their performance solely with the performance of high-skilled agents. Our policy analysis shows that, while positional high-skilled agents will attempt to perform too difficult tasks compared with the first-best outcome, the outcome for the low-skilled agents is ambiguous. If low-skilled agents choose to implement a self-protective strategy in the presence of interpersonal comparisons, they may end up attempting to perform less difficult tasks compared with the first-best outcome.

Our policy analysis suggests that a system of entrance fees, indexed to the difficulty level of the activity, can fully mitigate positional externalities. However, such a system is likely to be difficult to implement in practice. Another possibility is to link fees to rescue operations but since the assistance cost function is not able to target the agents' decisions perfectly, the optimal design of this function would not be a sufficient policy tool to implement the first-best outcome.

Our analysis builds on a stylized model of a complex phenomenon. We would therefore like to discuss some limitations, and possible directions for future research. First, our model only includes two ability types. If the social group instead would consist of three or more types, then the interpersonal comparisons may become more intricate. For example, the lowest ability type may not aspire to mimic the behavior of the highest ability type but to the middle type, or an average of the middle and high ability type. In these situations, corrective policy is potentially much harder to implement because of the informational burden required to assess the interpersonal comparison patterns. If the corrective policy cannot be based on actual assessments of how individuals make comparisons between themselves, but instead is based on some (maybe imperfect) estimate of these patterns, an interesting question for future research is to what degree corrective policy in such situations could lead to improvements in welfare.

Second, we assume that agents compare the difficulty of their attempted task with a weighted average of the difficulty levels chosen by other agents. Since the activity is risky for all agents, some proportion of attempted tasks will fail. An interesting question is therefore if agents compare with the attempted difficulty level among others, or if the success rate matters? ${ }^{16}$ Under incomplete information, positional agents have incentives to only display successful attempts (e.g., via social media). It is also possible that some agents send out false signals of success (e.g., a photo taken before giving up). This creates a bias in the informational flow, which forces agents to make guestimates of other agents' behavior. Since our recall is vulnerable to the availability heuristic (Tversky and Kahneman 1973), it is likely that agents will overestimate the frequency of successful attempts. This may affect agents' perception of both the success rate of others, and the objective risk associated with the activity. An interesting question for future researchers is therefore how policy interventions targeted at information about base frequencies and accident rates affect

$\overline{16}$ We are thankful for an anonymous reviewer for raising this question. 
risk-taking behavior among positional agents with different skill levels. A complicating factor for this type of research is that agents may both engage in strategic ignorance (e.g., Thunström et al. 2016) and fall pray for optimism bias (e.g., DeJoy 1989).

Third, research in psychology on social identity threat (e.g., Scheepers and Ellemers 2005; Scheepers et al. 2009) suggest that members of high-status groups experience negative affect and heightened stress levels when their status is is threatened by the relative performance of low-status individuals, i.e., when low-status individuals catch up. In our setting, this implies that high skilled agents may compare with low-skilled agents, if low-skilled perform at a level that is sufficiently close to the performance level of the high-skilled. A number of studies (see Collins 2000 for a review) further suggest that upward comparisons can, in some cases, be associated with increased wellbeing. Positive utility effects arise when low-status agents see the performance of high-status agents as a reflection of a possible future for themselves. Such perceptions are only credible if the difference in performance is small enough. Taken together, this suggests that the utility function related to relative status concerns is more complex than the simple functions commonly used by economists. Our analysis thus represent a special case and a stark simplification. For future research, it would be very interesting to evaluate how e.g., a non-linear cost-function for downward comparisons among the high-skilled, affect the analytical results.

\section{Appendix}

\section{Proof that $y^{i, \circ}>0$ when $\beta^{i}>0$}

To show that $y^{i, \circ}=0$ cannot be an optimal solution to the agent's maximization problem, let us evaluate the derivative $\tilde{U}_{y}^{i}$ at $y^{i}=0$

$$
\left.\tilde{U}_{y}^{i}\right|_{y^{i}=0}=\beta^{i} g_{y}(0)+\zeta^{i} I_{y}\left(0-\bar{y}^{i}\right)-v_{z}(1-h(0)) h_{y}(0)
$$

Here we have used $p^{i}(0)=1, g(0)=0$ and $I\left(0-\bar{y}^{i}\right)=I^{f}\left(0-\bar{y}^{i}\right)$ to simplify (21). Since the first term on the RHS approaches plus infinity (because $\lim _{y \rightarrow 0} g_{y}(y) \rightarrow \infty$ ), while the remaining terms on the RHS are finite, it follows that $\left.\tilde{U}_{y}^{i}\right|_{y^{i}=0}>0$. Hence, $y^{i}=0$ cannot satisfy the first-order condition $\tilde{U}_{y}^{i}=0$. Instead, the concavity of the objective function implies that the optimal choice features $y^{i, \circ}>0$ and $g^{i, \circ}=g\left(y^{i, \circ}\right)>0$. If the agent does not have preferences for personal pride, i.e. $\beta^{i}=0$, then $(21)$ reduces to

$$
\left.\tilde{U}_{y}^{i}\right|_{y^{i}=0}=\zeta^{i} I_{y}\left(\underset{>0}{\left(0-\bar{y}^{i}\right)}\right)-v_{z}\left(\underset{<0}{h(0))} h_{y}(0) .\right.
$$

In this special case, one cannot rule out that the two negative terms on the RHS in (22) are larger in absolute value, or equal to, the positive term on the RHS, in which case the agent would choose the corner $y^{i, \circ}=0$. 


\section{Proof that $y^{2, \circ}>y^{1, \circ}$ when the agents are identical in all aspects except in the probability of success}

When the two agent types are identical in all aspects except in the probability of success, then the low-skilled agent type's optimal choice $y^{1, \circ}$ satisfies the first-order condition in (4) for $\beta^{1}=\beta^{2}=\beta, \zeta^{1}=\zeta^{2}=\zeta$ and $\bar{y}^{1}=\bar{y}^{2}=\bar{y}$. Can the level $y^{1, \circ}$ also satisfy the corresponding first-order condition for the high-skilled type? To address this question, let us substitute $y^{2}=y^{1, \circ}$ into the expression for $\tilde{U}_{y}^{2}$. Then evaluate the difference $\left.\tilde{U}_{y}^{2}\right|_{y^{1, \circ}}-\left.\tilde{U}_{y}^{1}\right|_{y^{1, \circ}}$. We obtain

$$
\begin{aligned}
\left.\tilde{U}_{y}^{2}\right|_{y, \circ} & =\left[p^{2}\left(y^{1, \circ}\right)-p^{1}\left(y^{1, \circ}\right)\right]\left[\beta g_{y}\left(y^{1, \circ}\right)+\zeta I_{y}\left(y^{1, \circ}-\bar{y}\right)\right] \\
& +\left[p_{y}^{2}\left(y^{1, \circ}\right)-p_{y}^{1}\left(y^{1, \circ}\right)\right]\left[\beta g\left(y^{1, \circ}\right)+\zeta\left(I\left(y^{1, \circ}-\bar{y}\right)-I^{f}(0-\bar{y})\right)\right],
\end{aligned}
$$

where we on the LHS have used that $\left.\tilde{U}_{y}^{1}\right|_{y^{1, \circ}}=0$. From the properties of the probability function, it follows that $p^{2}\left(y^{1, \circ}\right)-p^{1}\left(y^{1, \circ}\right)>0$ and $p_{y}^{2}\left(y^{1, \circ}\right)-p_{y}^{1}\left(y^{1, \circ}\right)>0$. Hence, the expression on the RHS in (23) is positive. We therefore conclude that $\left.\tilde{U}_{y}^{2}\right|_{y^{1, \circ}}>0$ which, in turn implies that choosing $y^{2}=y^{1, \circ}$ cannot be an optimal solution for the high-skilled agent. Instead, the concavity of the objective function implies that the optimal choice of the high-skilled agent satisfies $y^{2, \circ}>y^{1, \circ}$.

\section{Examples when $\eta-\rho \varepsilon^{p}$ and $\varepsilon^{l}-\varepsilon^{p}$ are positive/negative at the optimum}

To exemplify when $\eta-\rho \varepsilon^{p}$ and $\varepsilon^{I}-\varepsilon^{p}$ are positive/negative at the private optimum, we use the following functional forms

$$
g(y)=y^{1-\gamma}, p(y)=1-y, I(y-\bar{y})=1-e^{-(y-\bar{y})}, v(z)=k z, h(y)=y,
$$

where we set $y^{\max }=1$. By using these functional forms, it follows that

$$
\eta=y, \rho=e^{y}-1, \varepsilon^{I}=\frac{y}{e^{y}-1}, \varepsilon^{p}=\frac{y}{1-y}
$$

We set $\beta=1, \zeta=0.01, \gamma=0.1$ and $\bar{y}=0.5$. The first-order condition in Eq. (4) can then be written as

$$
\tilde{U}_{y}=(1-y)\left[0.9 y^{-0.1}+0.01 e^{-(y-0.5)}\right]-\left[y^{0.9}+0.01\left(e^{0.5}-e^{-(y-0.5)}\right)\right]-k=0 .
$$

Equation (24) implicitly defines a negative relationship between the optimal choice $y^{\circ}$ and the marginal utility of leisure, $k$.

Let us first consider a value for $y^{\circ}$ such that $\eta>\rho \varepsilon^{p}$ and $\varepsilon^{I}>\varepsilon^{p}$ hold at the private optimum. This can be achieved if we, for example, set $k=0.6186120962351367$ in which case $y^{\circ} \approx 0.20$ satisfies (24). This implies 


$$
\begin{gathered}
\eta=0.2, \rho=e^{0.2}-1 \approx 0.22, \varepsilon^{I}=\frac{0.2}{e^{0.2}-1} \approx 0.90, \\
\varepsilon^{p}=\frac{0.2}{1-0.8}=0.25, \eta-\rho \varepsilon^{p}=0.145, \varepsilon^{I}-\varepsilon^{p}=0.65 .
\end{gathered}
$$

Hence, $\eta>\rho \varepsilon^{p}$ and $\varepsilon^{I}>\varepsilon^{p}$.

Next, let us consider a value for $y^{\circ}$ such that $\eta<\rho \varepsilon^{p}$ and $\varepsilon^{I}<\varepsilon^{p}$ hold at the private optimum. This can be achieved if we, for example, set $k=0.00682772130532$ in which case $y^{\circ} \approx 0.47$ satisfies (24). This implies

$$
\begin{aligned}
\eta & =0.47, \rho=e^{0.47}-1 \approx 0.59999, \varepsilon^{I}=\frac{0.47}{e^{0.47}-1} \approx 0.78334, \\
\varepsilon^{p} & =\frac{0.47}{1-0.47} \approx 0.88679, \eta-\rho \varepsilon^{p}=-0.06206513, \varepsilon^{I}-\varepsilon^{p}=-0.10345 .
\end{aligned}
$$

Hence, $\eta<\rho \varepsilon^{p}$ and $\varepsilon^{I}<\varepsilon^{p}$.

\section{The reaction functions and the myopic equilibrium}

Substituting $\bar{y}^{1}=x^{1} y^{1, \circ}+\left(1-x^{1}\right) y^{2}$ into Eq. (12) and differentiating w.r.t. $y^{1, \circ}$ and $y_{2}$ produces

$$
\frac{\partial y_{R F}^{1, \circ}}{\partial y^{2}}=-\left(1-x^{1}\right) \frac{p^{1} \zeta^{1} I_{y \bar{y}}^{1}+p_{y}^{1} \zeta^{1}\left(I_{\bar{y}}^{1}-I_{\bar{y}}^{1, f}\right)}{\tilde{U}_{y y}^{1}+x^{1}\left[p^{1} \zeta^{1} I_{y \bar{y}}^{1}+p_{y}^{1} \zeta^{1}\left(I_{\bar{y}}^{1}-I_{\bar{y}}^{1, f}\right)\right]} .
$$

Note that we can rearrange (6) to read $p^{1} \zeta^{1} I_{y \bar{y}}^{1}+p_{y}^{1} \zeta^{1}\left(I_{\bar{y}}^{1}-I_{\bar{y}}^{1, f}\right)=-\tilde{U}_{y y}^{1} \partial y^{1, \circ} / \partial \bar{y}^{1}$. Use this in (25)

$$
\frac{\partial y_{R F}^{1, \circ}}{\partial y^{2}}=-\left(1-x^{1}\right) \frac{\left(-\tilde{U}_{y y}^{1} \frac{\partial y^{1, \circ}}{\partial \bar{y}^{1}}\right)}{\tilde{U}_{y y}^{1}+x^{1}\left(-\tilde{U}_{y y}^{1} \frac{\partial y^{1, \circ}}{\partial \bar{y}^{1}}\right)}=\frac{1-x^{1}}{1-x^{1} \frac{\partial y^{1, \circ}}{\partial \bar{y}^{1}}} \frac{\partial y^{1, \circ}}{\partial \bar{y}^{1}} .
$$

The expression after the second equality in (26) is the first equation in (14). The second equation in (14) is derived analogously.

A sufficient condition for the existence of a myopic equilibrium with positively sloped reaction functions is that $\partial y^{i, \circ} / \partial \bar{y}^{i}<1$ holds for $i=1,2$. To show this, recall first that as long as $\beta^{i}>0$, then an agent's optimal choice features $y^{i, \circ}>0$. This implies that the intercept of $R F^{1}$ with the horizontal axis in $\left(y^{1}, y^{2}\right)$ space features $y^{1, \circ}>0$ while the intercept of $R F^{2}$ with the vertical axis in $\left(y^{1}, y^{2}\right)$ space features $y^{2, \circ}>0$. This is illustrated in Fig. 2 and implies that $R F^{2}$ is initially situated above $R F^{1}$ in $\left(y^{1}, y^{2}\right)$ space. If $R F^{2}$ has a flatter slope than $R F^{1}$ in $\left(y^{1}, y^{2}\right)$ space, the two functions will eventually intersect, as illustrated in Fig. 2, and this point is the myopic equilibrium. This implies that the myopic equilibrium will exist if the following two conditions are satisfied 


$$
\begin{gathered}
\frac{\partial y_{R F}^{1, \circ}}{\partial y^{2}}=\frac{1-x^{1}}{1-x^{1} \frac{\partial y^{1, \circ}}{\partial \bar{y}^{1}}} \frac{\partial y^{1, \circ}}{\partial \bar{y}^{1}}<1 \Rightarrow \frac{1}{\partial y_{R F}^{1, \circ} / \partial y^{2}}>1 \\
\frac{\partial y_{R F}^{2, \circ}}{\partial y^{1}}=\frac{1-x^{2}}{1-x^{2} \frac{\partial y^{2, \circ}}{\partial \bar{y}^{2}}} \frac{\partial y^{2, \circ}}{\partial \bar{y}^{2}}<1 .
\end{gathered}
$$

Note that the inequality in (28) is satisfied if $\partial y^{2, \circ} / \partial \bar{y}^{2}<1$, because then both the first term after the equality sign, $\left(1-x^{2}\right) /\left(1-x^{2} \partial y^{2, \circ} / \partial \bar{y}^{2}\right)$, and the second term, $\partial y^{2, \circ} / \partial \bar{y}^{2}$, are less than one. Therefore, also the product of these terms is less than one so that $\partial y_{R F}^{2, \circ} / \partial y^{1}<1$. A similar argument can be applied to show that the first inequality in (27) is satisfied if $\partial y^{1, \circ} / \partial \bar{y}^{1}<1$. Hence, if $\partial y^{i, \circ} / \partial \bar{y}^{i}<1$ holds for $i=1,2$, then $R F^{2}$ has a flatter slope than $R F^{1}$ in $\left(y^{1}, y^{2}\right)$ space and the myopic equilibrium exists.

\section{Derivation of Proposition 4}

We parameterize the entrance cost function as a fourth-order polynomial; $\varphi\left(y^{i}\right)=a_{1} y^{i}+a_{2}\left(y^{i}\right)^{2}+a_{3}\left(y^{i}\right)^{3}+a_{4}\left(y^{i}\right)^{4}$. The first-order conditions associated with the policy-maker's maximization problem become (after we have used the private first-order condition for each agent type to simplify the resulting expressions)

$$
\begin{aligned}
& \frac{\partial L}{\partial a_{1}}=\gamma\left[N^{1} y^{1}+N^{1} \varphi_{y}^{1} \frac{\partial y^{1}}{\partial a_{1}}+N^{2} y^{2}+N^{2} \varphi_{y}^{2} \frac{\partial y^{2}}{\partial a_{1}}\right]-N^{1} y^{1} u_{c}^{1}-N^{2} y^{2} u_{c}^{2}-\mu \frac{\partial y^{2}}{\partial a_{1}}=0 \\
& \frac{\partial L}{\partial a_{2}}=\gamma\left[N^{1}\left(y^{1}\right)^{2}+N^{1} \varphi_{y}^{1} \frac{\partial y^{1}}{\partial a_{2}}+N^{2}\left(y^{2}\right)^{2}+N^{2} \varphi_{y}^{2} \frac{\partial y^{2}}{\partial a_{2}}\right] \\
&-N^{1}\left(y^{1}\right)^{2} u_{c}^{1}-N^{2}\left(y^{2}\right)^{2} u_{c}^{2}-\mu \frac{\partial y^{2}}{\partial a_{2}}=0 \\
& \frac{\partial L}{\partial a_{3}=}=\gamma\left[N^{1}\left(y^{1}\right)^{3}+N^{1} \varphi_{y}^{1} \frac{\partial y^{1}}{\partial a_{3}}+N^{2}\left(y^{2}\right)^{3}+N^{2} \varphi_{y}^{2} \frac{\partial y^{2}}{\partial a_{3}}\right] \\
&-N^{1}\left(y^{1}\right)^{3} u_{c}^{1}-N^{2}\left(y^{2}\right)^{3} u_{c}^{2}-\mu \frac{\partial y^{2}}{\partial a_{3}}=0 \\
& \frac{\partial L}{\partial a_{4}}= \gamma\left[N^{1}\left(y^{1}\right)^{4}+N^{1} \varphi_{y}^{1} \frac{\partial y^{1}}{\partial a_{4}}+N^{2}\left(y^{2}\right)^{4}+N^{2} \varphi_{y}^{2} \frac{\partial y^{2}}{\partial a_{4}}\right] \\
&-N^{1}\left(y^{1}\right)^{4} u_{c}^{1}-N^{2}\left(y^{2}\right)^{4} u_{c}^{2}-\mu \frac{\partial y^{2}}{\partial a_{4}}=0
\end{aligned}
$$




$$
\frac{\partial L}{\partial \bar{y}}=\mu\left(1-\frac{\partial y^{2}}{\partial \bar{y}}\right)-\sum_{i} N^{i}\left[p^{i} \zeta^{i} I_{\bar{y}}^{i}+\left(1-p^{i}\right) \zeta^{i} I_{\bar{y}}^{i, f}\right]+\gamma\left(N^{1} \varphi_{y}^{1} \frac{\partial y^{1}}{\partial \bar{y}}+N^{2} \varphi_{y}^{2} \frac{\partial y^{2}}{\partial \bar{y}}\right)=0
$$

Dividing (29)-(32) by $\gamma$ and rearranging, produces the following equation system.

$$
\left[\begin{array}{cccc}
N^{1} y^{1} & N^{2} y^{2} & N^{1} \frac{\partial y^{1}}{\partial a_{1}} & N^{2} \frac{\partial y^{2}}{\partial a_{1}} \\
N^{1}\left(y^{1}\right)^{2} & N^{2}\left(y^{2}\right)^{2} & N^{1} \frac{\partial y^{1}}{\partial a_{2}} & N^{2} \frac{\partial y^{2}}{\partial a_{2}} \\
N^{1}\left(y^{1}\right)^{3} & N^{2}\left(y^{2}\right)^{3} & N^{1} \frac{\partial y^{1}}{\partial a_{3}} & N^{2} \frac{\partial y^{2}}{\partial a_{3}} \\
N^{1}\left(y^{1}\right)^{4} & N^{2}\left(y^{2}\right)^{4} & N^{1} \frac{\partial y^{1}}{\partial a_{4}} & N^{2} \frac{\partial y^{2}}{\partial a_{4}}
\end{array}\right]\left[\begin{array}{c}
1-\frac{u_{c}^{1}}{\gamma} \\
1-\frac{u_{c}^{2}}{\gamma} \\
\varphi_{y}^{1} \\
\varphi_{y}^{2}
\end{array}\right]=\left[\begin{array}{c}
\frac{\mu}{\gamma} \frac{\partial y^{2}}{\partial a_{1}} \\
\frac{\mu}{\gamma} \frac{\partial y^{2}}{\partial a_{2}} \\
\frac{\mu}{\gamma} \frac{\partial y^{2}}{\partial a_{3}} \\
\frac{\mu}{\gamma} \frac{\partial y^{2}}{\partial a_{4}}
\end{array}\right] .
$$

Applying Cramer's rule to solve for $1-u_{c}^{1} / \gamma$ produces

$$
1-\frac{u_{c}^{1}}{\gamma}=\frac{\left|\begin{array}{llll}
\frac{\mu}{\gamma} \frac{\partial y^{2}}{\partial a_{1}} & N^{2} y^{2} & N^{1} \frac{\partial y^{1}}{\partial a_{1}} & N^{2} \frac{\partial y^{2}}{\partial a_{1}} \\
\frac{\mu}{\gamma} \frac{\partial y^{2}}{\partial a_{2}} & N^{2}\left(y^{2}\right)^{2} & N^{1} \frac{\partial y^{1}}{\partial a_{2}} & N^{2} \frac{\partial y^{2}}{\partial a_{2}} \\
\frac{\mu}{\gamma} \frac{\partial y^{2}}{\partial a_{3}} & N^{2}\left(y^{2}\right)^{3} & N^{1} \frac{\partial y^{1}}{\partial a_{3}} & N^{2} \frac{\partial y^{2}}{\partial a_{3}} \\
\frac{\mu}{\gamma} \frac{\partial y^{2}}{\partial a_{4}} & N^{2}\left(y^{2}\right)^{4} & N^{1} \frac{\partial y^{1}}{\partial a_{4}} & N^{2} \frac{\partial y^{2}}{\partial a_{4}}
\end{array}\right|}{\left|\begin{array}{ccccc}
N^{1} y^{1} & N^{2} y^{2} & N^{1} \frac{\partial y^{1}}{\partial a_{1}} & N^{2} \frac{\partial y^{2}}{\partial a_{1}} \\
N^{1}\left(y^{1}\right)^{2} & N^{2}\left(y^{2}\right)^{2} & N^{1} \frac{\partial y^{1}}{\partial a_{2}} & N^{2} \frac{\partial y^{2}}{\partial a_{2}} \\
N^{1}\left(y^{1}\right)^{3} & N^{2}\left(y^{2}\right)^{3} & N^{1} \frac{\partial y^{1}}{\partial a_{3}} & N^{2} \frac{\partial y^{2}}{\partial a_{3}} \\
N^{1}\left(y^{1}\right)^{4} & N^{2}\left(y^{2}\right)^{4} & N^{1} \frac{\partial y^{1}}{\partial a_{4}} & N^{2} \frac{\partial y^{2}}{\partial a_{4}}
\end{array}\right| .}
$$

Since the first row in the determinant in the numerator is a linear function of the fourth row (multiplying each term in the fourth row by $\mu /\left(\gamma N^{2}\right)$ produces the first row), the determinant in the numerator is zero. Hence $u_{c}^{1}=\gamma$. Next, we apply Cramer's rule on (34) to solve for $1-u_{c}^{2} / \gamma$

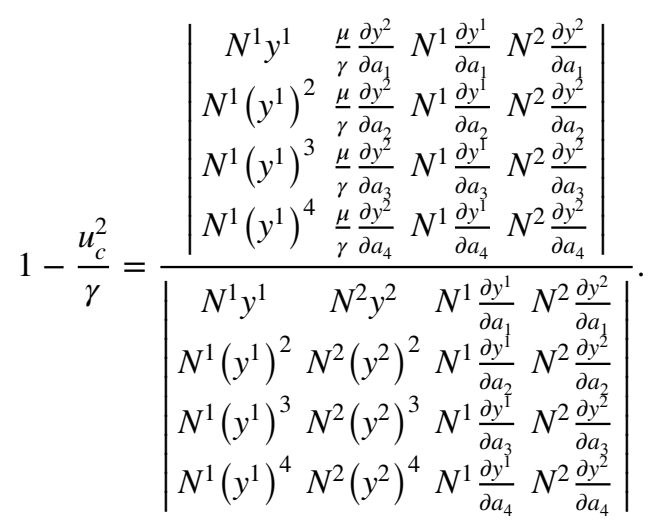


Since the second row in the determinant in the numerator is a linear function of the fourth row, the determinant in the numerator is zero. Hence $u_{c}^{2}=\gamma$. Applying Cramer's rule on (34) to solve for $\varphi_{y}^{1}$ gives

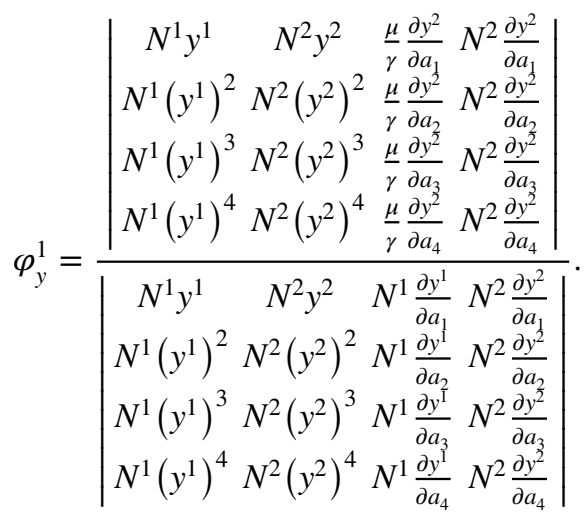

Since the third row in the determinant in the numerator is a linear function of the fourth row, the determinant in the numerator is zero. Hence $\varphi_{y}^{1}=0$. Finally, applying Cramer's rule on (34) to solve for $\varphi_{y}^{2}$ gives

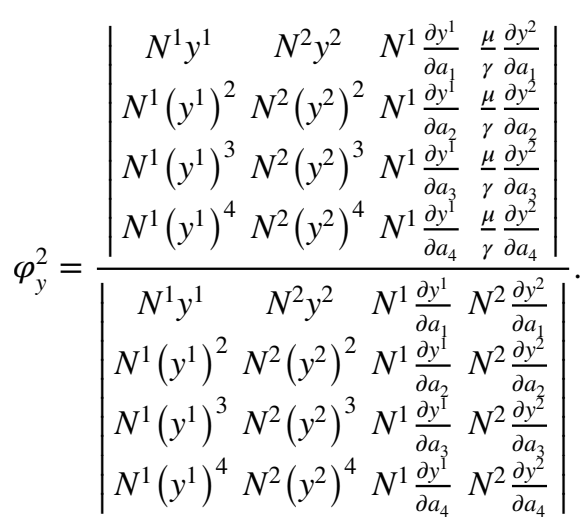

To evaluate the expression on the RHS in (38), we evaluate the determinant in the numerator (denoted $\left|\boldsymbol{H}_{1}\right|$ ) and the determinant in the denominator (denoted $|\boldsymbol{H}|$ ) by expanding along the fourth row for each determinant. This produces

$$
\begin{aligned}
& \left|\boldsymbol{H}_{1}\right|=\frac{\mu}{\gamma}\left[\frac{\partial y^{2}}{\partial a_{1}}(-1)^{5} C_{1}^{1}+\frac{\partial y^{2}}{\partial a_{2}}(-1)^{6} C_{1}^{2}+\frac{\partial y^{2}}{\partial a_{3}}(-1)^{7} C_{1}^{3}+\frac{\partial y^{2}}{\partial a_{4}}(-1)^{8} C_{1}^{4}\right], \\
& |\boldsymbol{H}|=N^{2}\left[\frac{\partial y^{2}}{\partial a_{1}}(-1)^{5} C_{1}^{1}+\frac{\partial y^{2}}{\partial a_{2}}(-1)^{6} C_{1}^{2}+\frac{\partial y^{2}}{\partial a_{3}}(-1)^{7} C_{1}^{3}+\frac{\partial y^{2}}{\partial a_{4}}(-1)^{8} C_{1}^{4}\right],
\end{aligned}
$$

where $C_{1}^{1}$ to $C_{1}^{4}$ are the cofactors associated with expanding along the fourth row. Note that these cofactors are the same for both $\left|\boldsymbol{H}_{1}\right|$ and $|\boldsymbol{H}|$. Substituting the expressions for $\left|\boldsymbol{H}_{1}\right|$ and $|\boldsymbol{H}|$ into (38) produces $\varphi_{y}^{2}=\mu /\left(\gamma N_{2}\right)$. 
Turning to the shadow price, we first substitute $\varphi_{y}^{1}=0$ and $\varphi_{y}^{2}=\mu /\left(\gamma N_{2}\right)$ into (33). Then we use the definition of $M W P_{\bar{y}, w}^{i}$ in Eq. (18) together with $u_{w}^{1}=u_{c}^{1}=\gamma$. Finally, we divide by $\gamma$ and rearrange. This produces $\mu / \gamma=\sum_{i} N^{i} M W P_{\bar{y}, w^{i}}$.

\section{Derivation of Proposition 5}

We parameterize the assistance cost function as a fourth-order polynomial; $\phi(y)=b_{1} y+b_{2} y^{2}+b_{3} y^{3}+b_{4} y^{4}$. The first-order conditions associated with the policy-maker's maximization problem become (after we have used the private firstorder condition for each agent type to simplify the resulting expressions)

$$
\begin{aligned}
& \frac{\partial L}{\partial b_{1}}=\gamma\left[\sum_{i} \tilde{N}^{i}\left(y^{i}+\left(\phi_{y}^{i}-C_{y}^{i}\right) \frac{\partial y^{i}}{\partial b_{1}}\right)+\sum_{i}\left(\phi^{i}-C^{i}\right) \tilde{N}_{y}^{i} \frac{\partial y^{i}}{\partial b_{1}}\right]-\sum_{i} \tilde{N}^{i} y^{i} u_{c}^{i, f}-\mu \frac{\partial y^{2}}{\partial b_{1}}=0, \\
& \frac{\partial L}{\partial b_{2}}=\gamma\left[\sum_{i} \tilde{N}^{i}\left(\left(y^{i}\right)^{2}+\left(\phi_{y}^{i}-C_{y}^{i}\right) \frac{\partial y^{i}}{\partial b_{2}}\right)+\sum_{i}\left(\phi^{i}-C^{i}\right) \tilde{N}_{y}^{i} \frac{\partial y^{i}}{\partial b_{2}}\right] \\
& -\sum_{i} \tilde{N}^{i}\left(y^{i}\right)^{2} u_{c}^{i, f}-\mu \frac{\partial y^{2}}{\partial b_{2}}=0 \text {, } \\
& \frac{\partial L}{\partial b_{3}}=\gamma\left[\sum_{i} \tilde{N}^{i}\left(\left(y^{i}\right)^{3}+\left(\phi_{y}^{i}-C_{y}^{i}\right) \frac{\partial y^{i}}{\partial b_{3}}\right)+\sum_{i}\left(\phi^{i}-C^{i}\right) \tilde{N}_{y}^{i} \frac{\partial y^{i}}{\partial b_{3}}\right] \\
& -\sum_{i} \tilde{N}^{i}\left(y^{i}\right)^{3} u_{c}^{i, f}-\mu \frac{\partial y^{2}}{\partial b_{3}}=0, \\
& \frac{\partial L}{\partial b_{4}}=\gamma\left[\sum_{i} \tilde{N}^{i}\left(\left(y^{i}\right)^{4}+\left(\phi_{y}^{i}-C_{y}^{i}\right) \frac{\partial y^{i}}{\partial b_{4}}\right)+\sum_{i}\left(\phi^{i}-C^{i}\right) \tilde{N}_{y}^{i} \frac{\partial y^{i}}{\partial b_{4}}\right] \\
& -\sum_{i} \tilde{N}^{i}\left(y^{i}\right)^{4} u_{c}^{i, f}-\mu \frac{\partial y^{2}}{\partial b_{4}}=0 \text {, } \\
& \frac{\partial L}{\partial \bar{y}}=\mu\left(1-\frac{\partial y^{2}}{\partial \bar{y}}\right)+\sum_{i} N^{i} \tilde{U}_{\bar{y}}^{i}+\gamma\left[\sum_{i} \tilde{N}^{i}\left(\phi_{y}^{i}-C_{y}^{i}\right) \frac{\partial y^{i}}{\partial \bar{y}}+\sum_{i}\left(\phi^{i}-C^{i}\right) \tilde{N}_{y}^{i} \frac{\partial y^{i}}{\partial \bar{y}}\right]=0,
\end{aligned}
$$

where $\tilde{N}_{y}^{i}=-\left[\left(1-p^{i}\right) d q^{i} / d y^{i}+\left(1-q^{i}\right) d p^{i} / d y^{i}\right] N^{i}>0$. Dividing Eqs. (41)-(44) by $\gamma$ and rearranging, produces the following equation system 


$$
\left[\begin{array}{cccc}
\tilde{N}^{1} y^{1} & \tilde{N}^{2} y^{2} & \tilde{N}^{1} \frac{\partial y^{1}}{\partial b_{1}} & \tilde{N}^{2} \frac{\partial y^{2}}{\partial b_{1}} \\
\tilde{N}^{1}\left(y^{1}\right)^{2} & \tilde{N}^{2}\left(y^{2}\right)^{2} & \tilde{N}^{1} \frac{\partial y}{\partial b_{2}} & \tilde{N}^{2} \frac{\partial y^{y_{2}}}{\partial b_{2}} \\
\tilde{N}^{1}\left(y^{1}\right)^{3} & \tilde{N}^{2}\left(y^{2}\right)^{3} & \tilde{N}^{1} \frac{\partial y^{1}}{\partial b_{3}} & \tilde{N}^{2} \frac{\partial y^{2}}{\partial b_{3}} \\
\tilde{N}^{1}\left(y^{1}\right)^{4} & \tilde{N}^{2}\left(y^{2}\right)^{4} & \tilde{N}^{1} \frac{\partial y^{1}}{\partial b_{4}} & \tilde{N}^{2} \frac{y^{2}}{\partial b_{4}}
\end{array}\right]\left[\begin{array}{c}
1-\frac{u_{c}^{1, f}}{\gamma} \\
1-\frac{u_{c}^{2}}{\gamma} \\
\gamma \\
\left(\phi_{y}^{1}-C_{y}^{1}+\frac{\left(\phi^{1}-C^{1}\right)}{\tilde{N}^{1}} \tilde{N}_{y}^{1}\right) \\
\left(\phi_{y}^{2}-C_{y}^{2}+\frac{\left(\phi^{2}-C^{2}\right)}{\tilde{N}^{2}} \tilde{N}_{y}^{2}\right)
\end{array}\right]=\left\lceil\begin{array}{c}
\frac{\mu}{\gamma} \frac{\partial y^{2}}{\partial b_{1}} \\
\frac{\mu}{\gamma} \frac{\partial y^{2}}{\partial b_{2}} \\
\frac{\mu}{\gamma} \frac{\partial y^{2}}{\partial b_{3}} \\
\frac{\mu}{\gamma} \frac{\partial y^{2}}{\partial b_{4}}
\end{array}\right]
$$

Applying Cramer's rule to solve for to solve for $1-u_{c}^{1, f} / \gamma$ produces

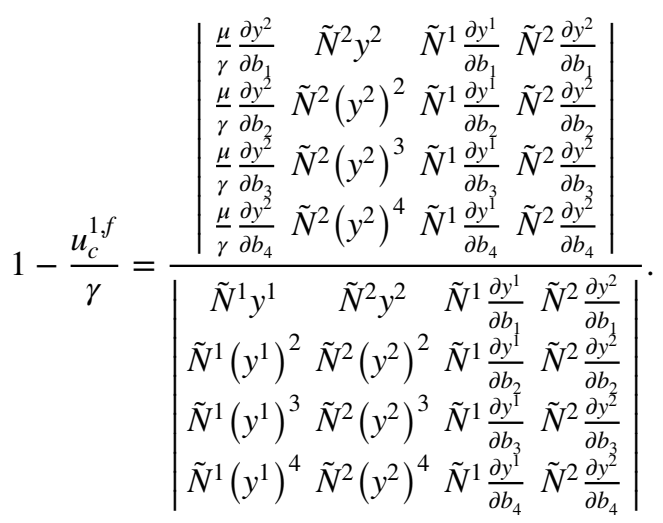

Since the first row in the determinant in the numerator is a linear function of the fourth row (multiplying each term in the fourth row by $\mu /\left(\gamma \tilde{N}^{2}\right)$ produces the first row), the determinant in the numerator is zero. Hence $u_{c}^{1, f}=\gamma$. Next, we apply Cramer's rule on (46) to solve for $1-u_{c}^{2, f} / \gamma$

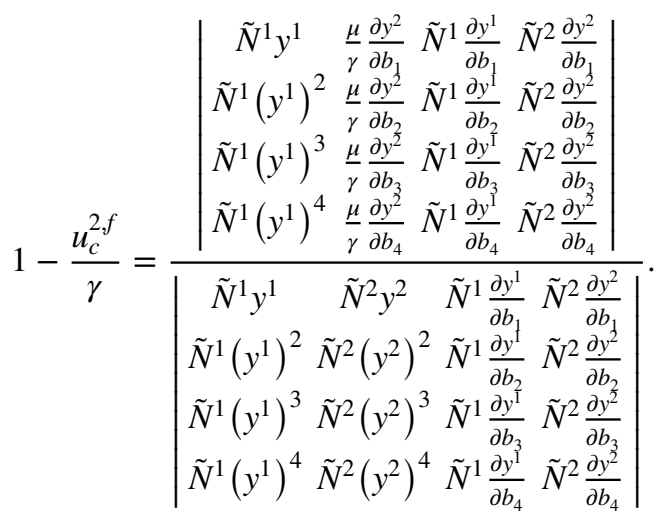

Since the second row in the determinant in the numerator is a linear function of the fourth row, the determinant in the numerator is zero. Hence $u_{c}^{2, f}=\gamma$. Furthermore 


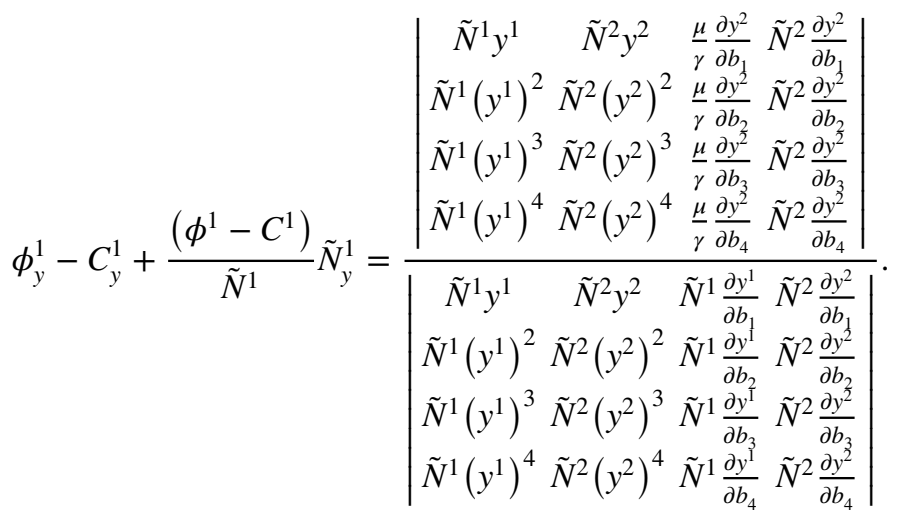

Since the third row in the determinant in the numerator is a linear function of the fourth row, the determinant in the numerator is zero. Hence $\phi_{y}^{1}=C_{y}^{1}-\left(\phi^{1}-C^{1}\right) \tilde{N}_{y}^{1} / \tilde{N}^{1}$. Finally

$$
\phi_{y}^{2}-C_{y}^{2}+\frac{\left(\phi^{2}-C^{2}\right)}{\tilde{N}^{2}} \tilde{N}_{y}^{2}=\frac{\left|\begin{array}{cccc}
\tilde{N}^{1} y^{1} & \tilde{N}^{2} y^{2} & \tilde{N}^{1} \frac{\partial y^{1}}{\partial b_{1}} & \frac{\mu}{\gamma} \frac{\partial y^{2}}{\partial b_{1}} \\
\tilde{N}^{1}\left(y^{1}\right)^{2} & \tilde{N}^{2}\left(y^{2}\right)^{2} & \tilde{N}^{1} \frac{\partial y^{1}}{\partial b_{2}} & \frac{\mu}{\gamma} \frac{\partial y^{2}}{\partial b_{2}} \\
\tilde{N}^{1}\left(y^{1}\right)^{3} & \tilde{N}^{2}\left(y^{2}\right)^{3} & \tilde{N}^{1} \frac{\partial y^{1}}{\partial b_{3}} & \frac{\mu}{\gamma} \frac{\partial y^{2}}{\partial b_{3}} \\
\tilde{N}^{1}\left(y^{1}\right)^{4} & \tilde{N}^{2}\left(y^{2}\right)^{4} & \tilde{N}^{1} \frac{\partial y^{1}}{\partial b_{4}} & \frac{\mu}{\gamma} \frac{\partial y^{2}}{\partial b_{4}}
\end{array}\right|}{\left|\begin{array}{cccc}
\tilde{N}^{1} y^{1} & \tilde{N}^{2} y^{2} & \tilde{N}^{1} \frac{\partial y^{1}}{\partial b_{1}} & \tilde{N}^{2} \frac{\partial y^{2}}{\partial b_{1}} \\
\tilde{N}^{1}\left(y^{1}\right)^{2} & \tilde{N}^{2}\left(y^{2}\right)^{2} & \tilde{N}^{1} \frac{\partial y^{1}}{\partial b_{2}} & \tilde{N}^{2} \frac{\partial y^{2}}{\partial b_{2}} \\
\tilde{N}^{1}\left(y^{1}\right)^{3} & \tilde{N}^{2}\left(y^{2}\right)^{3} & \tilde{N}^{1} \frac{\partial y^{1}}{\partial b_{3}} & \tilde{N}^{2} \frac{\partial y^{2}}{\partial b_{3}} \\
\tilde{N}^{1}\left(y^{1}\right)^{4} & \tilde{N}^{2}\left(y^{2}\right)^{4} & \tilde{N}^{1} \frac{\partial y^{1}}{\partial b_{4}} & \tilde{N}^{2} \frac{\partial y^{2}}{\partial b_{4}}
\end{array}\right|}
$$

To evaluate the expression on the RHS, we evaluate the determinant in the numerator (denoted $\left|\boldsymbol{H}_{1}\right|$ ) and the determinant in the denominator (denoted $|\boldsymbol{H}|$ ) by expanding along the fourth row for each determinant. This produces

$$
\begin{aligned}
& \left|\boldsymbol{H}_{1}\right|=\frac{\mu}{\gamma}\left[\frac{\partial y^{2}}{\partial b_{1}}(-1)^{5} C_{1}^{1}+\frac{\partial y^{2}}{\partial b_{2}}(-1)^{6} C_{1}^{2}+\frac{\partial y^{2}}{\partial b_{3}}(-1)^{7} C_{1}^{3}+\frac{\partial y^{2}}{\partial b_{4}}(-1)^{8} C_{1}^{4}\right], \\
& |\boldsymbol{H}|=\widehat{N}_{2}\left[\frac{\partial y^{2}}{\partial b_{1}}(-1)^{5} C_{1}^{1}+\frac{\partial y^{2}}{\partial b_{2}}(-1)^{6} C_{1}^{2}+\frac{\partial y^{2}}{\partial b_{3}}(-1)^{7} C_{1}^{3}+\frac{\partial y^{2}}{\partial b_{4}}(-1)^{8} C_{1}^{4}\right],
\end{aligned}
$$

where $C_{1}^{1}$ to $C_{1}^{4}$ are the cofactors associated with expanding along the fourth row. Note that these cofactors are the same for both $\left|\boldsymbol{H}_{1}\right|$ and $|\boldsymbol{H}|$. Substituting the expressions for $\left|\boldsymbol{H}_{1}\right|$ and $|\boldsymbol{H}|$ into (500) and rearranging produces $\phi_{y}^{2}=C_{y}^{2}-\left(\phi^{2}-C^{2}\right) \tilde{N}_{y}^{2} / \tilde{N}^{2}+\mu /\left(\gamma \tilde{N}^{2}\right)$. Turning to the shadow price, first rewrite Eq. (18) to read $\tilde{U}_{\bar{y}}^{i}=-\tilde{U}_{w}^{i} M W P_{\bar{y}, w}^{i}$. Using this expression, together with the equa- 
tions defining $\phi_{y}^{1}$ and $\phi_{y}^{2}$, produces $\mu=\sum_{i} N^{i} M W P_{\bar{y}, w}^{i} \tilde{U}_{w}^{i}$. Dividing by $\gamma$, and using the definition of $\pi^{i}$ together with $\gamma=u_{w}^{1, f}=u_{w}^{2, f}$, produces $\mu / \gamma=\sum_{i} \pi^{i} N_{i} M W P_{\bar{y}, w}^{i}$.

Acknowledgements We are grateful for helpful comments and suggestions by Professor Derek Clark, and for financial funding from the Norwegian Research Council, Research Grant dnr: 262626.

Funding Open Access funding provided by UiT The Arctic University of Norway.

Open Access This article is licensed under a Creative Commons Attribution 4.0 International License, which permits use, sharing, adaptation, distribution and reproduction in any medium or format, as long as you give appropriate credit to the original author(s) and the source, provide a link to the Creative Commons licence, and indicate if changes were made. The images or other third party material in this article are included in the article's Creative Commons licence, unless indicated otherwise in a credit line to the material. If material is not included in the article's Creative Commons licence and your intended use is not permitted by statutory regulation or exceeds the permitted use, you will need to obtain permission directly from the copyright holder. To view a copy of this licence, visit http://creativecommons.org/licen ses/by/4.0/.

\section{References}

Akerlof GA, Kranton RE (2000) Economics and Identity. Q J Econ 115:715-753. https://doi.org/10.1162/ 003355300554881

Aloise-Young PA, Hennigan KM, Graham JW (1996) Role of the self-image and smoker stereotype in smoking onset during early adolescence: a longitudinal study. Health Psychol 15(6):494-497. https://doi.org/10.1037/0278-6133.15.6.494

Alpizar F, Carlsson F, Johansson-Stenman O (2005) How much do we care about absolute versus relative income and consumption? J Econ Behav Organ 56:405-421. https://doi.org/10.1016/j.jebo.2002.10. 007

Aronsson T, Johansson-Stenman O (2008) When the Joneses' consumption hurts: optimal public good provision and nonlinear income taxation. J Public Econ 92:986-997. https://doi.org/10.1016/j.jpube co.2007.12.007

Aronsson T, Johansson-Stenman O (2010) Positional concerns in an OLG model: optimal labor and capital income taxation. Int Econ Rev 51:1071-1095. https://doi.org/10.1111/j.1468-2354.2010.00611.x

Bakshi GS, Chen Z (1996) The Spirit of Capitalism and Stock Market Prices. Am Econ Rev 86 (1): 133-157. www.jstor.org/stable/2118259

Baumeister RF, Tice DM, Hutton DG (1989) Self-presentational motivations and personality differences in self-esteem. J Pers 57:547-579. https://doi.org/10.1111/j.1467-6494.1989.tb02384.x

Blanchflower DG, Oswald AJ (2004) Well-Being over time in Britain and the USA. J Public Econ 88:1359-1386. https://doi.org/10.1016/S0047-2727(02)00168-8

Boone JL (1998) The evolution of magnanimity. Human Nature 9:1-21. https://doi.org/10.1007/ s12110-998-1009-y

Burke PJ, Reitzes DC (1981) The Link Between Identity and Role Performance. Soc Psychol Q 44: 83-92. www.jstor.org/stable/3033704

Burke PJ (1991) Identity Processes and Social Stress. Am Sociol Rev 56: 836-849. www.jstor.org/stable/ 2096259

Clark AE, Senik C (2010) Who Compares to Whom? The Anatomy of Income Comparisons in Europe. The Economic Journal 120(544):573-594. https://doi.org/10.1111/j.1468-0297.2010.02359.x

Carlsson F, Johansson-Stenman O, Martinsson P (2007) Do you enjoy having more than others? Survey evidence of positional goods. Economica 74:586-598. https://doi.org/10.1111/j.1468-0335.2006. 00571.x

Chang W-C (2013) Climbing up the social ladders: identity, relative income and subjective well-being. Soc Indic Res 113(1):513-535. https://doi.org/10.1007/s11205-012-0108-7 
Collins RL (2000) Among the better ones: Upward assimilation in social comparison. In: Suls J, Wheeler L (eds) Handbook of socialcomparison. Kluwer Academic/Plenum, New York, pp 159-172. https:// doi.org/10.1007/978-1-4615-4237-7_9

Corneo G, Jeanne O (1997) Snobs, bandwagons, and the origin of social customs in consumer behavior. J Econ Behav Organ 32:333-347. https://doi.org/10.1016/S0167-2681(96)00024-8

Cowan R, Cowan W, Swann P (2004) Waves in consumption with interdependence among consumers. Can J Econ 37:149-177. https://doi.org/10.1111/j.0008-4085.2004.008_1.x

Dejoy DM (1989) The optimism bias and traffic accident risk perception. Accid Anal Prev 21(4):333340. https://doi.org/10.1016/0001-4575(89)90024-9

Duesenberry JS (1949) Income, saving and the theory of consumer behavior. Harvard University Press, Cambridge (ISBN-13: 978-0674447509)

Dupor B, Liu WF (2003) Jealousy and equilibrium overconsumption. Am Econ Rev 93:423-428. https:// doi.org/10.1257/000282803321455395

Easterlin RA (1995) Will raising the incomes of all increase the happiness of all? J Econ Behav Organ 27:35-47. https://doi.org/10.1016/0167-2681(95)00003-B

Eckerstorfer P, Wendner R (2013) Asymmetric and non-atmospheric consumption externalities, and efficient consumption taxation. J Public Econ 106:42-56. https://doi.org/10.1016/j.jpubeco.2013.07. 003

Ferrer-i-Carbonell A (2005) Income and well-being: an empirical analysis of the comparison income effect. J Public Econ 89:997-1019. https://doi.org/10.1016/j.jpubeco.2004.06.003

Festinger L (1957) A theory of cognitive dissonance. Stanford University Press, Stanford (ISBN978-0-8047-0911-8)

Fischer P, Vingilis E, Greitemeyer T, Vogrinic C (2011) Risk-taking and the media. Risk Anal 31(5):699_ 705. https://doi.org/10.1111/j.1539-6924.2010.01538.x

Fleissbach K, Weber B, Trautner P, Dohmen T, Sunde U et al (2007) Social comparison affects rewardrelated brain activity in the human ventral striatum. Science 318:1305-1308. https://doi.org/10. 1126/science. 1145876

Grafen A (1990a) Sexual selection unhandicapped by the fisher process. J Theor Biol 144(4):473-516. https://doi.org/10.1016/S0022-5193(05)80088-8

Grafen A (1990b) Bilogical signals as handicaps. J Theor Biol 144(4):517-546. https://doi.org/10.1016/ S0022-5193(05)80087-6

Hogg MA (2016) Social Identity Theory. In: McKeown S, Haji R, Ferguson N (eds) Understanding peace and conflict through social identity theory. Peace psychology book series. Springer, Cham, pp 3-17. https://doi.org/10.1007/978-3-319-29869-6_1

Jellison JM, Riskind J (1970) A social comparison of abilities interpretation of risk-taking behavior. J Pers Soc Psychol 15(4):375-390. https://doi.org/10.1037/h0029601

Johansson-Stenman O, Carlsson OF, Daruvala D, Martinsson P (2006) Honestly, why are you driving a BMW? J Econ Behav Organ 60:129-146. https://doi.org/10.1016/j.jebo.2004.08.006

Kirkpatrick LA, Ellis BJ (2007) An evolutionary-psychological approach to self-esteem: multiple domains and multiple functions. In: Brewer MB, Hewstone M (eds) Perspectives on social psychology. Self and social identity. Blackwell Publishing, Malden, pp 52-77. https://doi.org/10.1002/ 9780470998557.ch16

Leary MR, Tchividijian LR, Kraxberger BE (1994) Self-presentation can be hazardous to your health: impression management aaloisend health risk. Health Psychol 13(6):461-470. https://doi.org/10. 1037/0278-6133.13.6.461

Ljungqvist L, Uhlig H (2000) Tax policy and aggregate demand management under catching up with the Joneses. Am Econ Rev 90:356-366. https://doi.org/10.1257/aer.90.3.356

Luttmer EFP (2005) Neighbors as negatives: relative earnings and well-being. Q J Econ 120:963-1002. https://doi.org/10.1093/qje/120.3.963

Miller-Johnson S, Costanzo PR, Coie JD, Rose MR, Browne DC, Johnson C (2003) Peer social structure and risk-taking behaviors among African American Early Adolescents. J Youth Adolesc 32:375384. https://doi.org/10.1023/A:1024926132419

Mujcic R, Frijters P (2013) Economic choices and status: measuring preferences for income rank. Oxf Econ Pap 65(1):47-73. https://doi.org/10.1093/oep/gpr065

Rivis A, Sheeran P (2003) Descriptive norms as an additional predictor in the theory of planned behaviour: a meta-analysis. Curr Psychol 22(3):2018-2233. https://doi.org/10.1007/s12144-003-1018-2 
Scheepers D, Ellemers N (2005) When the pressure is up: the assessment of social identity threat in low and high status groups. J Exp Soc Psychol 41(2):192-200. https://doi.org/10.1016/j.jesp.2004.06. 002

Scheepers D, Ellemers N, Sintemaartensdijk N (2009) Suffering from the possibility of status loss: physiological responses to social identity threat in high status groups. Eur J Soc Psychol 39(6):10751092. https://doi.org/10.1002/ejsp.609

Solnick S, Hemenway D (1998) Is more always better? a survey on positional concerns. J Econ Behav Organ 37:373-383. https://doi.org/10.1016/S0167-2681(98)00089-4

Solnick S, Hemenway D (2005) Are positional concerns stronger in some domains that in others? Am Econ Rev Pap Proc 95:147-151. https://doi.org/10.1257/000282805774669925

Stets JE, Burke PJ (2000) Identity theory and social identity theory. Soc Psychol Q 63(3):224-237. https://doi.org/10.2307/2695870

Stryker S (1968) Identity salience and role performance. J Marriage Fam 4:558-564. https://doi.org/10. $2307 / 349494$

Stryker S, Serpe RT (1982) Commitment, identity salience, and role behavior: a theory and research example. In: Ickes W, Knowles ES (eds) Personality, roles, and social behavior. Springer, New York, 199 - 218. https://doi.org/10.1007/978-1-4613-9469-3_7

Tajfel H (1982) Social identity and intergroup relations. Cambridge University Press, Cambridge (ISBN: $\mathbf{9 7 8 0 5 2 1 1 5 3 6 5 2 )}$

Tajfel H, Billig M, Bundy RP, Flament C (1971) Social categorization and intergroup behaviour. Eur J Soc Psychol 1:149-177. https://doi.org/10.1002/ejsp.2420010202

Techel F, Jarry F, Kronthaler G, Mitterer S, Nairz P, Pavšek M, Valt M, Darms G (2016) Avalanche fatalities in the European Alps: long-term trends and statistics. Geographica Helvetica 71:147-159. https://doi.org/10.5194/gh-71-147-2016

Thunström L, Nordström J, Shogren JF, Ehmnke M, van’tVeld K (2016) Strategic self-ignorance. J Risk Uncertain 52:117-136. https://doi.org/10.1007/s11166-016-9236-9

Turner J, Oakes P (1986) The significance of the social identity concept for social psychology with reference to individualism, interactionism and social influence. Br J Soc Psychol 25(3):237-252. https:// doi.org/10.1111/j.2044-8309.1986.tb00732.x

Tversky A, Kahneman D (1973) Availability: a heuristic for judging frequency and probability. Cogn Psychol 5(2):207-232. https://doi.org/10.1016/0010-0285(73)90033-9

Veblen T [1994](1899) The Theory of the Leisure Class. Penguin twentieth-century classics. Introduction by Robert Lekachman. Penguin Books, New York. ISBN 978-0-14-018795-3

White KM, Smith JR, Terry DJ, Greenslade JH, McKimmie BM (2009) Social influence in the theory of planned behaviour: the role of descriptive, injunctive, and in-group norms. Br J Soc Psychol 48(1):135-158. https://doi.org/10.1348/014466608X295207

Wolfe RN, Lennox RD, Cutler BL (1986) Getting along and getting ahead: empirical support for a theory of protective and acquisitive self-presentation. J Pers Soc Psychol 50:356-361. https://doi.org/10. 1037/0022-3514.50.2.356

Zahavi A (1975) Mate selection—a selection for a handicap. J Theor Biol 53(1):2305-3214. https://doi. org/10.1016/0022-5193(75)90111-3

Publisher's Note Springer Nature remains neutral with regard to jurisdictional claims in published maps and institutional affiliations. 